ARTICLE

DOI: $10.1038 / \mathrm{s} 41467-018-03921-6$

\title{
A B-ARR-mediated cytokinin transcriptional network directs hormone cross-regulation and shoot development
}

\author{
Mingtang Xie ${ }^{1,2}$, Hongyu Chen ${ }^{3}$, Ling Huang ${ }^{4}$, Ryan C. O'Neil ${ }^{1,5}$, Maxim N. Shokhirev ${ }^{4}$ \& Joseph R. Ecker ${ }^{1,2}$
}

Cytokinin fulfills its diverse roles in planta through a series of transcriptional responses. We identify the in vivo DNA binding site profiles for three genetically redundant type-B ARABIDOPSIS RESPONSE REGULATORS (B-ARRs): ARR1, ARR10, and ARR12. The expression and genome-wide DNA binding locations of the three $B$-ARRs extensively overlap. Constructing a primary cytokinin response transcriptional network reveals a recurring theme of widespread cross-regulation between the components of the cytokinin pathway and other plant hormone pathways. The B-ARRs are found to have similar DNA binding motifs, though sequences flanking the core motif were degenerate. Cytokinin treatments amalgamate the three different B-ARRs motifs to identical DNA binding signatures (AGATHY, $\mathrm{H}(\mathrm{a} / \mathrm{t} / \mathrm{c}$ ), $\mathrm{Y}(\mathrm{t} / \mathrm{c})$ ) which suggests cytokinin may regulate binding activity of B-ARR family members. Furthermore, we find that WUSCHEL, a key gene required for apical meristem maintenance, is a cytokinin-dependent B-ARR target gene, demonstrating the importance of the cytokinin transcription factor network in shoot development.

\footnotetext{
${ }^{1}$ Plant Biology Laboratory, and Genomic Analysis Laboratory, The Salk Institute for Biological Studies, La Jolla, CA 92037, USA. ${ }^{2}$ Howard Hughes Medical Institute, The Salk Institute for Biological Studies, La Jolla, CA 92037, USA. ${ }^{3}$ Department of Computer Science, Dartmouth College, Hanover, NH 03755, USA. ${ }^{4}$ The Razavi Newman Integrative Genomics and Bioinformatics Core Facility, The Salk Institute for Biological Studies, La Jolla, CA 92037, USA.

${ }^{5}$ Bioinformatics Program, University of California at San Diego, La Jolla, CA 92093, USA. Correspondence and requests for materials should be addressed to J.R.E. (email: ecker@salk.edu)
} 
C ytokinin, an $N^{6}$-substituted adenine derivative, along with other phytohormones orchestrates almost every aspect of plant growth and development, including meristem function, vascular development, biotic and abiotic stresses, and leaf senescence ${ }^{1-5}$. Cytokinin was first discovered for its ability to promote cell division over fifty years ago ${ }^{6}$. In the past twenty years, its own biosynthesis and signaling pathways and diverse roles in regulating cellular processes have been revealed by both forward and reverse genetic screens $s^{2,7-12}$. Cytokinin employs a two-component multi-step phosphorelay for its perception and signaling transduction ${ }^{12-14}$. In Arabidopsis, there are three cytokinin receptors (ARABIDOPSIS HISTIDINE KINASEs; $A H K 2,3,4)$ and eleven type-B response regulators (ARABIDOSPIS RESPONSE REGULATORs; $B$-ARRs) ${ }^{8,15}$. In cytokinin signaling cascades, the histidine-containing phosphor-transfer proteins (AHPs) act as phosphor-transfer intermediates for various AHK-AHP-B-ARR modules ${ }^{16}$. Genetic analysis also revealed that only higher order mutants of each family render pronounced developmental phenotypes, indicating redundancy in the cytokinin signaling pathway ${ }^{8}$.

The cytokinin transcriptional response centrally affects the family of ARRs. Type-B ARRs (B-ARRs) are transcription factors (TFs) with a GARP-like DNA binding domain at their C-termini and a receiver domain at their $\mathrm{N}$-termini. Type-A ARRs (AARRs) are similar to the N-termini receiver domain of B-ARRs but do not possess a DNA binding domain. A-ARRs are negative cytokinin regulators but their mechanism of inhibition in cytokinin signaling remains unknown ${ }^{12}$. The DNA binding domain and protein nuclear localization signal domain at the C-terminus of B-ARRs are responsible for B-ARRs entering the nucleus and binding to their targets while their activation domain is responsible for the activation of cytokinin transcriptional responses. The presence of the receiver domain in B-ARRs is thought to cause inhibition at low cytokinin levels and may block the upstream phosphorelay to B-ARRs. It is postulated that the receiver domain masks the DNA binding domain of B-ARRs until its conformation is altered by cytokinin, which finally results in the activation of $\mathrm{B}-\mathrm{ARRs}^{15}$. In previous genetic analyses, five Arabidopsis BARRs were shown to act in cytokinin signaling cascades with ARR1, ARR10, and ARR12 playing critical roles in plant growth and development ${ }^{8}$. The $A-A R R s$ are cytokinin response genes that are the targets of B-ARR TFs ${ }^{12}$. However, the $B$-ARRs are not regulated at the transcriptional level by cytokinin but are post transcriptionally controlled ${ }^{11}$. Recently, B-ARRs were shown to be regulated at the level of protein stability, at least in part, through the ubiquitin-proteasome pathway ${ }^{17}$.

Previous in vitro studies have identified candidate binding motifs for the B-ARRs ${ }^{18,19}$ and a "golden list" of cytokinin response genes from microarray expression data and RNA-seq data $^{20}$. However, the identity of which cytokinin responsive genes may be direct targets of the B-ARRs remains unknown. In addition, most experiments have depended upon treatment with high concentrations of cytokinin, since the targets of B-ARRs are almost impossible to identify at the endogenous levels of cytokinin in transcriptomic studies. Therefore, identification of the genome-wide targets of B-ARRs, with and without cytokinin treatment, would facilitate our understanding of the cytokinin responsive DNA regulatory elements, provide insights into cytokinin primary responsive gene expression, and potentially elucidate the mechanism(s) by which cytokinin ultimately regulates diverse physiological responses. Recently, genome-wide binding sites of ARR10 were identified by chromatin immunoprecipitation sequencing (ChIP-seq) of a tagged, over-expressed ARR10 fusion protein ${ }^{21}$, demonstrating the utility of in vivo DNA binding studies for cytokinin response pathway analysis.
Cytokinin plays an important but poorly understood role in the maintenance of the stem cell niche and regulation of meristem $\operatorname{size}^{22,23}$. First, inhibition of a subset of $A-A R R s$ by WUSCHEL (WUS) has been demonstrated, although the mechanism of this repression remains unknown ${ }^{24}$. Since $A-A R R s$ are targets of $\mathrm{B}$ ARRs, it can be postulated that the repression of $A-A R R s$ by WUS involves $B$-ARRs. Second, the arr1/10/12 triple mutant was shown to produce a smaller size shoot apical meristem ${ }^{8}$. Third, genetic manipulation of cytokinin levels either by loss-of-function mutants of LONELY GUYS, which are involved in the one step conversion of cytokinin precursors to active cytokinin ${ }^{25}$, or by over-expression of cytokinin oxidase produces meristem defects $^{26}$. Finally, plant regeneration requires the proper ratio of cytokinin and auxin ${ }^{27}$. Therefore, a greater understanding of the targets of B-ARRs may provide a link between the cytokinin transcriptional response and important plant developmental processes such as meristem development.

Our study aims to construct a core cytokinin transcriptional response network with a focus on systematic identification of the binding targets of the B-ARRs, the key players in cytokinin signaling. We take advantage of the power of recombineering ${ }^{28}$ to generate plants containing epitope-tagged $B$-ARRs and employ ChIP-seq to identify their genome-wide binding locations. Extensive targeting of multiple type-B ARRs to a common set of genes reveals a conserved core cytokinin transcriptional response network and extensive cross-regulation of the plant hormone pathways. We also demonstrate that the regulation of WUS by BARRs is critical for stem cell maintenance in the shoot apical meristem. These findings provide potential avenues to further explore the mechanism operating downstream cytokinin responses that control diverse growth and development processes.

\section{Results}

The protein localization of B-ARRs reveals extensive overlap. Previous genetic studies of the arr1/10/12 triple mutant revealed pronounced developmental phenotypes, such as smaller size seedling and adult plants, effects likely due to a smaller shoot apical meristem and insensitivity to cytokinin treatment ${ }^{2,8}$. Such studies revealed that ARR1, ARR10, ARR12 are critical components of the cytokinin signaling pathway (Fig. 1a). To explore the cellular distribution pattern of these three B-ARRs, Ypet (yellow fluorescent protein)-tagged B-ARRs lines were generated using a recombineering-based gene tagging technique ${ }^{28}$. The advantages of this strategy are that both the expression pattern and protein location can be monitored. Moreover, because the near-by gene (cis-) regulatory information is maintained, these tagged gene constructs provide a state nearest to the native expression of the endogenous B-ARRs as is currently technically possible in plants $^{28}$. ARR1, ARR10, and ARR12 tagged lines were generated in the Col-0 background and were used for ChIP-seq experiments. The functionality of these constructs was also confirmed by successful complementation of the arr1/10/12 triple mutant phenotype (Fig. 1b).

Previous organ-specific expression analysis using reverse transcription PCR and promoter reporter analysis using GUS staining revealed an overlapping expression pattern of BARRs ${ }^{8,29}$. We used our ARR-recombineering lines to study the expression pattern of B-ARRs in three-days-old seedlings in the absence and presence of cytokinin $(10 \mu \mathrm{M}$ 6-Benzylaminopurine (6-BA)). We found that all three B-ARRs had similar pattern expressed in roots, hypocotyl, and cotyledons (Fig. $1 \mathrm{c} ; n=3$ for each ARR gene). Consistent with previous findings, all three BARRs were localized in the nucleus ${ }^{18,29-31}$. However, the localization of ARR1 upon 6-BA treatment was more obvious in root tip and cotyledons than in other tissues (Fig. 1c-ARR1 + 

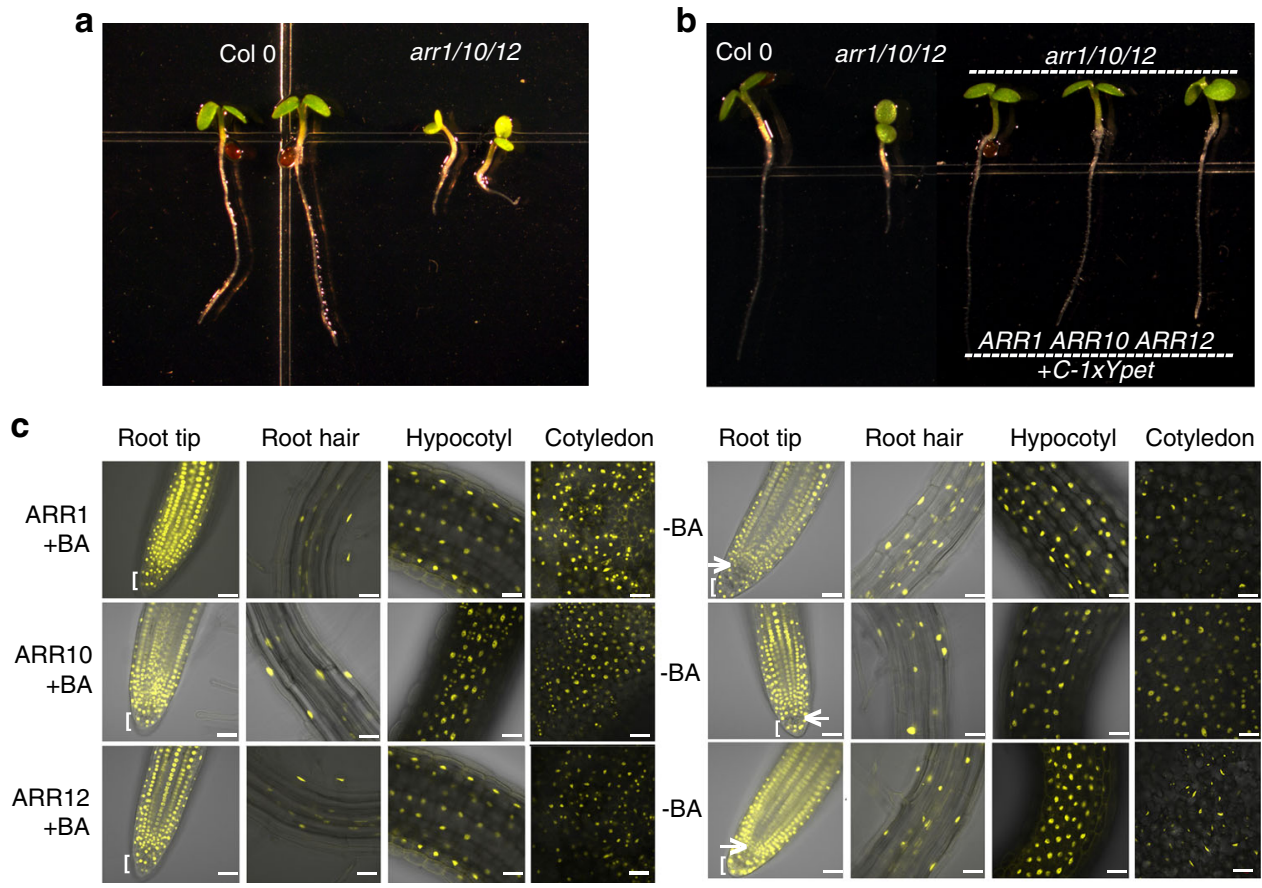

Fig. 1 B-ARRs protein localization upon cytokinin treatment. a The phenotype of 3-days-old arr1/10/12 triple mutants grown under long day (16-h light/8-h dark) conditions on MS. b Recombineering B-ARRs with Ypet at C-termini ( + C-1xYpet) can rescue arr1/10/12 triple mutant phenotypes. c The protein localization of B-ARRs in plants after 4-h treatments of $10 \mu \mathrm{M} 6-\mathrm{BA}(+\mathrm{BA})$ and DMSO ( $-\mathrm{BA})$. Three individual plants for each factor were imaged and representative images were presented $(n=3)$. Images of root tip regions (Root tip), root hair regions (Root hair), hypocotyls (Hypocotyl), and the adaxial side of cotyledons (Cotyledon) are shown. Arrows indicate the quiescent center. Square brackets indicate the columella cells. Scale bar $=40 \mu M$

BA). In contrast, the 6-BA treatment had less impact on the localization of ARR10 compared to other tested-ARRs (Fig. 1cARR10). This was consistent with previous findings identifying $\mathrm{ARR} 10$ as the most stable B-ARR ${ }^{8,32}$. Additionally, the intensity of ARR12 was slightly lower than the mock treatment in the root hair region of roots (Fig. 1c-ARR12-BA). One apparent discrepancy with previous results ${ }^{8,29}$ was the expression of ARR1, ARR10, ARR12 in columella cells of root tips (Fig. 1c, square bracket).

Construction of a cytokinin network using B-ARRs targets. The targets (genes near the DNA binding sites of B-ARRs) of three key B-ARRs $(1,10,12)$ were identified by ChIP-seq using long-day (16-h light $/ 8 \mathrm{~h}$ dark cycle) conditions at $22^{\circ} \mathrm{C}$ and 3 days-old seedlings growing vertically on plates containing Murashige and Skoog (MS) medium (Fig. 2a, and Supplementary Fig. 1, Supplementary Data 1 ). The binding profiles of all three factors were generated either in the absence of 6-BA (endogenous level of cytokinin) or in the presence of $10 \mu \mathrm{M}$ 6-BA (cytokinin treatment) for $4 \mathrm{~h}$, or 3 days (only for ARR1). Without 6-BA treatment, 2815 (ARR1_m), 4822 (ARR10_m), and 823 (ARR12_m) targets were identified using a cutoff of $p$-value 1E16 (MACS2 peak caller, cutoff: $+/-1.5 \mathrm{~kb}$ of genes). Using the same standards, the cytokinin treated samples had higher numbers of targets for all three B-ARRs (5128 (ARR1_BA), 6272 (ARR10_BA), and 6240 (ARR12_BA)). An increase in the number of targets upon cytokinin treatment might result from either protein stabilization or modification by phosphorylation ${ }^{11}$ or both processes. Interestingly, samples treated with $10 \mu \mathrm{M}$ 6-BA for three days showed even further increase in the number of targets for ARR1, up to approximately 10,000. B-ARR binding sites detected by ChIP-seq were highly enriched near gene transcription start sites (TSS) (Fig. 2b and Supplementary Fig. 2). They were enriched in regions $1.5 \mathrm{~kb}$ upstream and $1 \mathrm{~kb}$ downstream of genes, but enrichment dropped dramatically beyond $1 \mathrm{~kb}$ downstream of genes.

From comparison of genes near in vivo DNA binding sites for these B-ARRs at endogenous and elevated cytokinin levels, a TFTF interaction network was constructed to analyze the redundant and diverged role of B-ARR family members (Fig. 2c). In addition, a directed gene regulatory network was constructed using the three cytokinin-treated ChIP-Seq results and publicly available DAP-Seq results ${ }^{33}$ as edges and changes in target gene expression (as measured by steady state RNA level) as nodes (Fig. 2d). While the B-ARRs are not themselves transcriptionally cytokinin responsive, 6-BA induced an increase in B-ARR binding of cytokinin "target genes" suggesting they regulate the expression of cytokinin responsive genes ${ }^{11}$. Individual B-ARR shared many targets between mock and 6-BA treatment datasets. For example, $85 \%$ (4075 of 4822 mock treated plants) of ARR10 mock targets overlap with $4 \mathrm{~h}$ 6-BA targets (Fig. 2c). All $10 \mathrm{~A}$ ARRs (ARRs 3, 4, 5, 6, 7, 8, 9, 15, 16, and 17), well-known cytokinin response genes, were among the targets of B-ARRs (Fig. 3a). At endogenous cytokinin levels, ARR1, ARR10, and ARR12 shared 503 targets (Fig. 3b). In addition, ARR1 and ARR10 shared another 1915 targets. In contrast, ARR12 shared less targets than either ARR1 or ARR10. At the elevated cytokinin levels (4-h 6-BA treatment), a set of 3373 targets were shared by ARR1, ARR10, and ARR12 ("common set of targets") while 8770 targets were bounded by at least one of the three B-ARRs ("union set of targets") (Fig. 3c, Supplementary Data 2). Additionally, ARR1 shared more targets with ARR10 than ARR12. The results indicated that many targets under mock treatment (endogenous cytokinin) were also bound by B-ARRs upon 6-BA treatments of $4 \mathrm{~h}$ or 3 days (Fig. 3d). However, the number of target genes increased from 2815, 5128, to 10,340 up 6-BA treatments (Figs. 2c and $3 \mathrm{~d}$ ). When genome-wide binding sites of recombineered ARR10_BA were compared to those generated using ChIP-seq of a tagged, over-expressing ARR10 by Zubo and colleagues ${ }^{21}$, an 
a

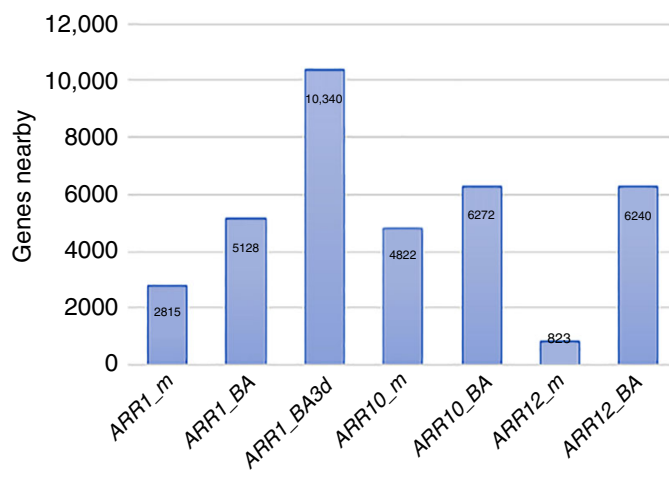

C

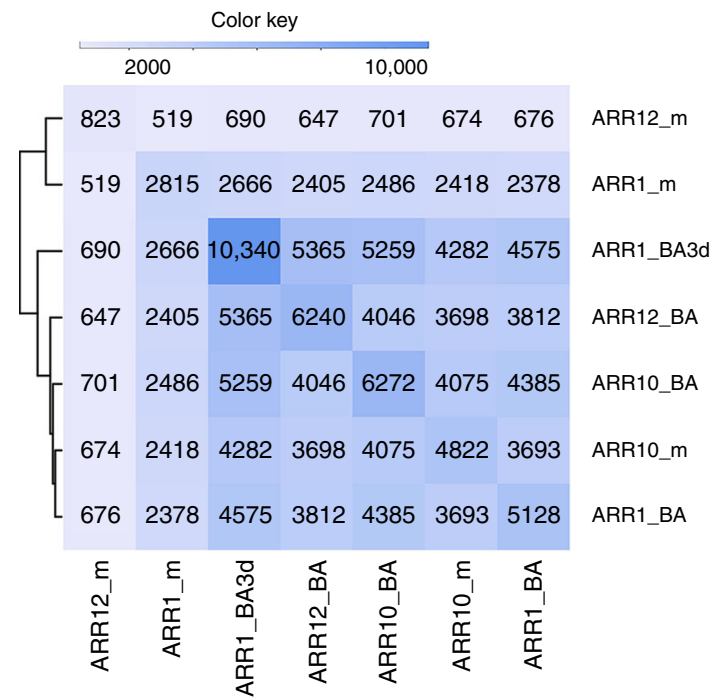

b

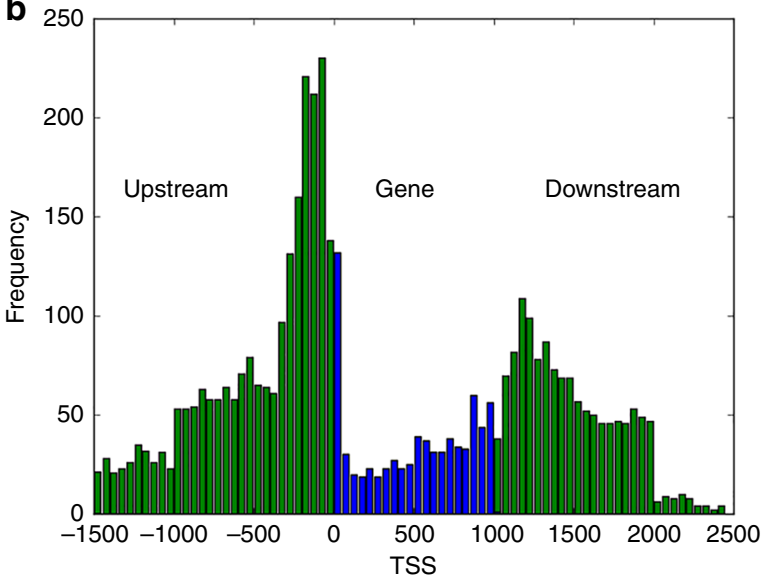

Summit location relative to TSS (bp)

d

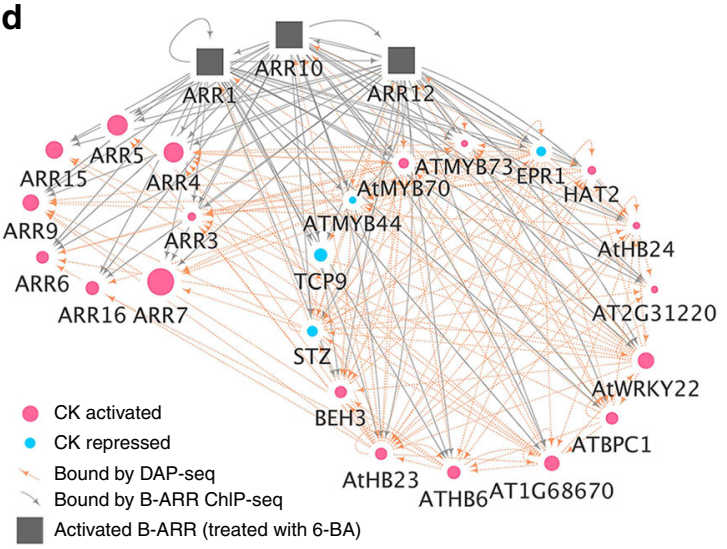

Fig. 2 Cytokinin-dependent transcriptional response network. a Potential target genes near the binding sites of B-ARRs by different treatments (mock treatment $(\mathrm{m})$, treatment with $10 \mu \mathrm{M}$ 6-BA for $4 \mathrm{~h}$ (BA), or treatment for 3 days (BA3d)). Nearby genes were determined by B-ARR binding within $1.5 \mathrm{~kb}$ upstream and downstream of the gene's annotation. The total number of nearby genes for each treatment is printed on each bar. $\mathbf{b}$ Distribution of ChIP-seq peaks around a normalized gene (1 kb) for ARR1_m. The base position relative to the transcription start site (TSS). c Heatmap showing number of the shared target genes of the B-ARRs TF-TF interaction network. Conditions are: mock treatment: ARR1_m, ARR10_m, ARR12_m; 4-h 10 $\mu$ M 6-BA treatment: ARR1_BA, ARR12_BA; 3-day $10 \mu \mathrm{M}$ 6-BA treatment: ARR1_BA3d. d A sub-network graph of the B-ARRs gene regulatory network. Nodes were either type-A ARRs or transcription factors from the core target genes of B-ARRs with DAP-seq data available. The sizes of nodes are in proportion to the max peak score of B-ARRs (Supplementary Data 5, column B). Nodes in red color are cytokinin activated while nodes in blue are cytokinin repressed. Edges in gray represent B-ARR direct bound genes and edges in salmon red represent DAP-seq bound genes. The position of nodes does not mean anything. This is the sub-network with the top 50\% ranked transcription factors and the entire directed network of B-ARR regulatory network was available as Supplementary Data 6

overall correlated profile of peak locations was found (Supplementary Fig. 3a). Compared to dataset 1 of Zubo et al. ${ }^{21} 2783$ $(69.5 \%)$ out of 4004 potential targets were also identified in our study (Fisher's Exact test, $p$-value $<0.001$, Supplementary Fig. 3b). If only the ARR10 "regulated targets" (those with evidence of transcriptional activity) in dataset 2 of Zubo's study were considered, $87.4 \%$ were overlapping with our B-ARR union target set (Supplementary Fig. 3c). Though the recombineering experiment and the over-expressing experiment showed a high degree of agreement, our combined analysis of three B-ARRs revealed many additional in planta B-ARRs genomic binding locations (Supplementary Fig. 3d). The gene expression analyses were done using RNA-seq data from plants treated with 6-BA treatment for $4 \mathrm{~h}$ and using an arr1/10/12 triple mutant (Supplementary Data 3 and Data 4). In total, 554 genes were differentially expressed in response to cytokinin treatment whereas the expression of 2323 genes were affected in the triple mutant ( $q$-value $<=0.05$ and 1.6-fold changes as cutoff, Supplementary Fig. 4a, b). When previous transcriptomic data and recent CaMV $35 S$ over-expression ARR10 ChIP-seq data ${ }^{21}$ were compared, the expression of 813 common targets and 1713 union targets were observed to be affected by cytokinin treatment (Supplementary Data 5). These union target genes that showed cytokinin-induced expression changes were designated as "core target genes" of the B-ARRs. The top 50 genes ranked by their maximum peak scores contained eight known cytokinin biosynthesis/degradation or response genes (16\%) including type- $A$ ARRs (ARR4, 5, 7,15), cytokinin receptor (WOL/AtHK4), and cytokinin degradation enzyme (CKX5) and showing a 10-fold enrichment $(p<0.001$, binomial test, Supplementary Fig. 5a, 5b). Finally, the intersection of 1713 core target genes and a previous large-scale TF binding dataset ${ }^{33}$ was used to construct a cytokinin 
a
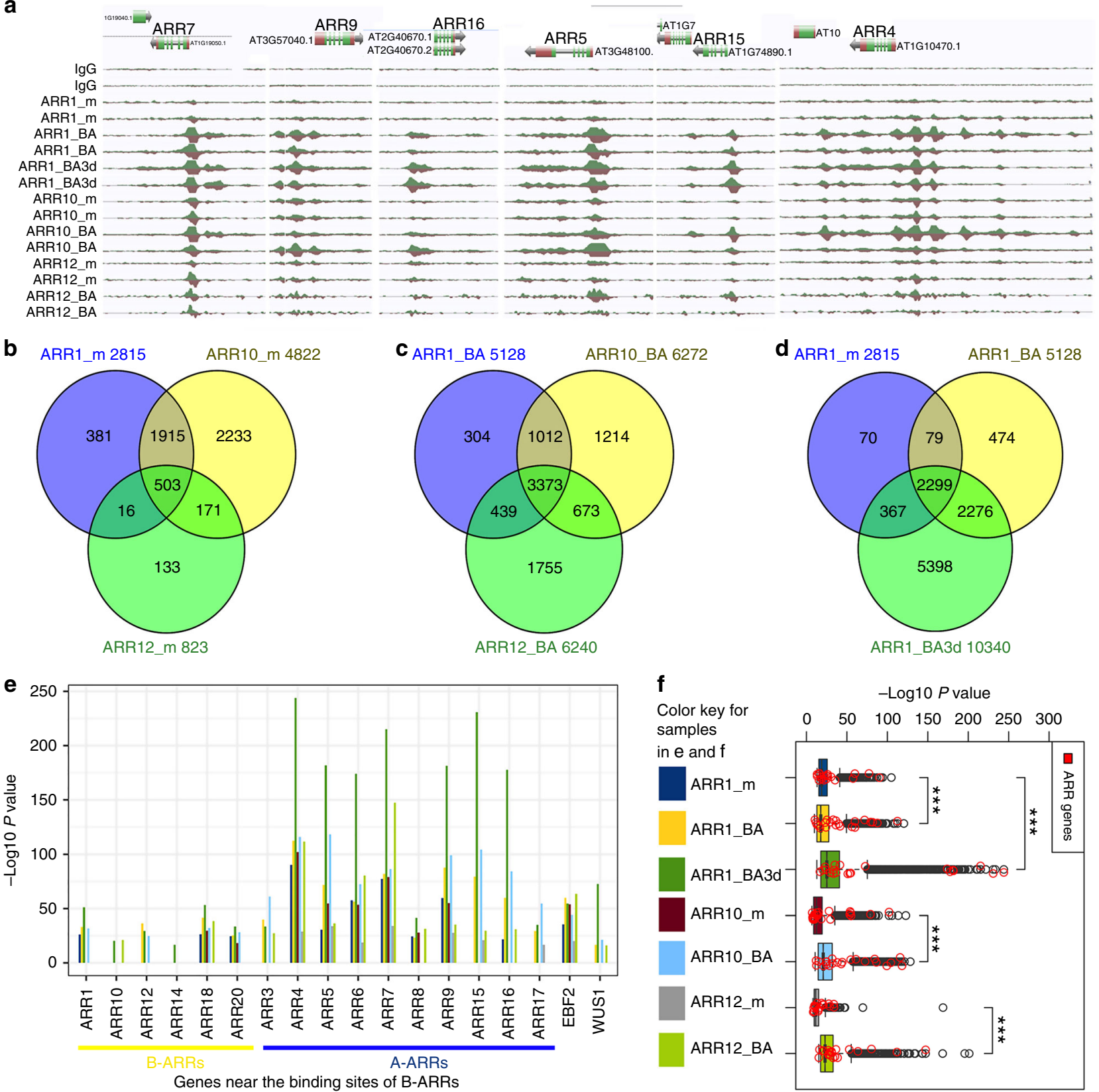

f

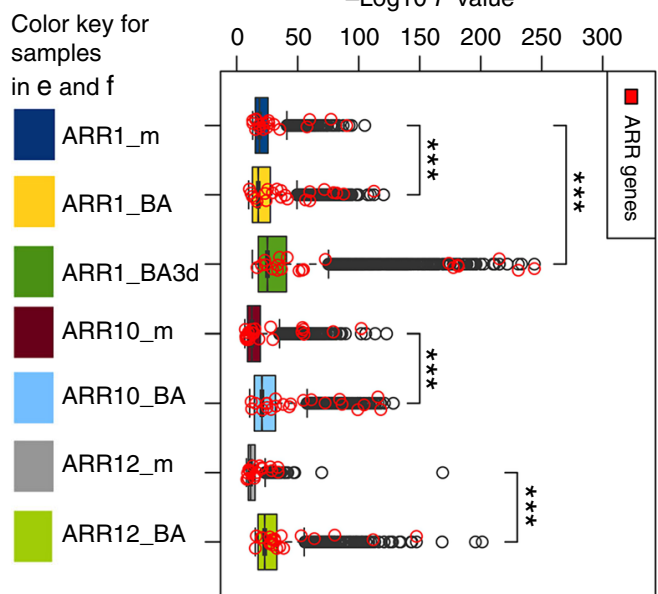

Fig. 3 A-ARRs are direct targets of B-ARR transcription factors. a AnnoJ browser snapshots of A-ARR genes, targets of B-ARR TFs. b Venn diagram describing the number of target genes for each of three key B-ARRs in the mock treatment $(\mathrm{m})$. $\mathbf{c}$ Venn diagram describing the number of target genes for each of three key B-ARRs in the $10 \mu \mathrm{M}$ 6-BA 4-h treatment (BA). d Venn diagram describing the number of target genes increase for ARR1 in a cytokinin time course treatment, $0 \mathrm{~h}(\mathrm{~m}), 4 \mathrm{~h}$ (BA), 3 days (BA3d). e Peak scores (-Log10 p-value) of ARRs in various cytokinin treatments. $\mathbf{f}$ Box plot showing the distributions of the binding peak scores of B-ARR targets. ARRs were highlighted in red circles. Asterisks $\left(^{\star \star \star}\right)$ indicates significant difference using Wilcoxon rank-sum test $(p<0.001)$

transcriptional gene regulatory network (Fig. 2d, Supplementary Data 6), providing a framework for future studies of cytokinin response genes.

A negative feedback loop in the cytokinin regulatory network. Previous indirect evidences including genetic studies, transcriptional profiling results, and promoter deletion analyses of $A-A R R s$ suggested that these genes are targets of B-ARRs ${ }^{20,34,35}$. Our results show significant increase of B-ARR binding to the promoter of $A-A R R s$ in response to cytokinin (Fig. 3a) and $A-A R R$ genes are among the top-ranking targets of multiple B-ARRs (percentile ranking $<5 \%$ ). Upon exogenous cytokinin treatment (6-BA treatment for $4 \mathrm{~h}$ to 3 days), B-ARRs show significant increase of binding to $A$-ARRs (Fig. $3 \mathrm{e}$ ) and their downstream target genes (Fig. 3f; Wilcoxon rank-sum test $p<0.001)$. In contrast, there was not much change in binding at the promoter of the EIN3 binding factor (EBF2) (Fig. 3e), a negative regulator in the ethylene signaling pathways ${ }^{36}$. Interestingly, a few $B$-ARRs, including ARR1, ARR10, ARR12, ARR18, and $A R R 14$ were among low-ranking targets (Fig. 3e). Overall these analyses support a scenario in which the promoters of the $A-A R R$ genes are bound by B-ARRs representing an efficient feedback mechanism to fine-tune cytokinin responses in the plant. 
Functional classification of B-ARRs targets. A previous study of binding sites for the master transcriptional regulator for the plant hormone ethylene revealed major feedback loops where EIN3 directly targeted almost all essential genes in the ethylene signaling pathway, as well as key regulators of other phytohormone pathways ${ }^{36}$. To find out whether this TF-governed auto-regulation and cross-regulation with other pathways also holds true or not for B-ARRs, we performed gene ontology (GO) analysis of target genes of B-ARRs using the top 3000 genes ranked by IDR score $^{3 p}$. These analyses revealed enrichment for similar biological function and processes for all tested B-ARRs (Supplementary Fig. 6). GO enrichment analysis was consistent with the fundamental and diverse role of cytokinin (Supplementary Fig. 6). BARR DNA binding sites are highly associated with plant hormone responsive genes and cytokinin genes (GOTERMs: response to plant hormone stimulus $p$-value $1.5 \mathrm{E}-26$, two-component signal transduction $p$-value 6.3E-15). Among the 3373 common targets of the three B-ARRs (ARR1/ARR10/ARR12) tested, we observed enrichment of hormone-related genes (Fig. 4a, Supplementary Data 7). Additionally, the top 3000 genes ranked by ARR10 peak scores were used to refine the GO analysis. The top GO terms are similar in the global analysis for the targets of three B-ARRs. These targets of B-ARRs include the primary cytokinin response genes, A-ARRs and the cytokinin receptor AHK4. Although BARRs are not regulated at the transcriptional level by cytokinin, it is interesting that several (ARR1, ARR10, ARR12, ARR14, and $A R R 18)$ were found in the B-ARR target gene list as being under control of B-ARRs. In addition, B-ARRs were found to bind at cytokinin biosynthesis and degradation pathway genes (Fig. 4a, Supplementary Data 7). Thus, the transcriptional responses directed by B-ARRs may include nearly every step from cytokinin perception and signaling transductions to the TFs. Like EIN3, the response of B-ARRs to cytokinin may involve cross-regulation with other plant hormone biosynthesis, signaling, and response pathways (Fig. 4a). B-ARRs were found to target the auxin receptor genes TIR1 and AFB2, as well as the auxin transportation efflux carrier genes, the Pin-formed and Pin-formed like (PIN3/4/ 7), and the GH3s genes which mediate auxin conjugation. Auxin transcriptional response regulators, including several ARFs and many $A u x / I A A s$ genes, were also among the list of B-ARR targets (Fig. 4a, Supplementary Data 7). The most striking features among the hormone-related targets of B-ARRs are genes encoding the master transcriptional factors such as MYC2, PIFs, BES/ $B Z R$, and ERFs (Fig. 4a), each responsible for mediating the transcriptional responses to other plant hormones ${ }^{38-42}$. B-ARRs also target genes encoding plant hormone receptors such as BRI1/ $B A K 1 / B A K 7^{43}$, PYR/PYLS $s^{4}$, and TIR1/AFB2 ${ }^{45}$ (Fig. 4a). In addition, $\mathrm{B}$-ARRs targeted the plant hormone negative signaling component genes, such as Aux/IAAs for auxin ${ }^{46}, E B F 1 / 2$ for ethylene $^{47}$, BIN2 for brassinosteroid ${ }^{43,48}$, and the DELLA protein $G A I$ and $R G A$ for gibberellin ${ }^{49}$.

Next, we inquired into whether the transcription of B-ARR target genes are regulated by cytokinin levels (Supplementary Data 3 and Data 4). The regulated genes were then layered into plant hormone pathways targeted by B-ARRs (Supplementary Data 7). Cytokinin treatment increased gene transcripts for most $A$-ARRs, AHK4, and cytokinin degradation enzymes in the cytokinin pathways (Supplementary Data 7). Whereas gene transcripts were shown to decrease for important negative regulators of other hormone pathways, including several IAAs, and GH3s for auxin, SnRK3.14 and NRT for abscisic acid (ABA), as well as modification enzyme for salicylic acid (SA) (Supplementary Data 7). In addition, in the triple mutant background, the auxin receptor AFB2 and the auxin efflux carrier PIN7 were down-regulated (Supplementary Data 7, gene names underscored) whereas TAS3 and the ethylene receptor ETR2 were up- regulated (Supplementary Data 7, gene names in green). These results suggest that cytokinin pathway TFs target important regulators of other plant hormones pathways, potentially leading to diverse outputs for developmental and growth programs, as well as responses to environmental cues.

Numerous studies have reported that TF binding does not necessarily coincide with changes in gene transcription ${ }^{50}$. In the case of EIN3, only $30 \%$ of the ET-induced binding events were associated with transcriptional changes ${ }^{36}$. We examined genes in the "golden list" for cytokinin responses which were identified by Bhargava $^{20}$ using a meta-analysis of multiple expression datasets, to determine the overlap with genes that we identified as B-ARR targets. In all, $116(73.4 \%)$ of 158 up-regulated genes in the golden list were identified among the 8770 union binding targets of three B-ARRs, suggesting significant association between BARR binding and cytokinin-induced transcriptional responses (Fig. 4b; Fisher's Exact Test $p<0.001$ ). When compared to the 3373 common targets of three B-ARRs, the percentage of overlap dropped to $35.4 \%$ which is still highly significant (Fig. 4b; Fisher's Exact Test $p<0.001)$. Similarly, 35 (51.4\%) of 68 down-regulated genes in the golden list were overlapped among the 8770 union targets of B-ARRs (Fisher's Exact Test $p<0.001$ ) although only $26.5 \%$ of these were among the 3373 common targets of the three key B-ARRs (Fig. 4b; Fisher's Exact Test $p<0.001$ ). A small portion (74 of 226 or 32.7\%) of genes in the golden list were not found in the list of B-ARRs targets. They might be either indirect cytokinin response genes or the targets of other B-ARRs that were not tested in this experiment. It is interesting that activators like B-ARRs directly target cytokinin repressed genes which may involve the recruitment of other co-regulators. Only $490(14.5 \%)$ of 3373 common targets of three B-ARRs (ARR1/ARR10/ARR12) were affected transcriptionally either in triple mutant or by cytokinin treatment (Fig. 4c). A subset of 162 common targets that the binding of B-ARRs responded to cytokinin treatment also changed their expression upon cytokinin treatment (Fig. 4d, Supplementary Fig. 4c). When another dataset of cytokinin response genes was used ${ }^{1}, 82 \%$ of overlap between the expressed gene list and our ChIP-seq data were observed (Fisher's Exact Test, $p<0.001)$.

Switch of B-ARR motif specificity in response to cytokinin. The targets of B-ARRs are predicted to be enriched for specific binding site motifs. We used the top 1000 conservative narrow peaks from each B-ARR, ranked by the IDR peak score and included two hundred bps flanking the peak summits, for motif calling using the MEME-ChIP suite (Fig. 5). Analyses of the identified B-ARRs motifs suggested several important conclusions. First, without any treatment, B-ARRs binding motifs are very similar to each other; each contains an AGAT core flanked by degenerate sequences with the first $\mathrm{A}$ in the core being slightly more degenerate $(\mathrm{A} / \mathrm{G})$. Although the motifs are similar, there are also significant differences that can be distinguished by the degenerate sequences (Fig. 5). These findings are consistent with previous in vitro binding and the PBM assays, identifying AGAT as the core B-ARR binding motif ${ }^{2}$. Second, after cytokinin treatment, all binding motifs strikingly become invariant AGAT $((t / a / c)(t / c))$ which we have termed the B-ARR-6-BA motif (AGATHY) (Fig. 5). In order to better understand the 6-BAdependent change of the core binding motif, we additionally analyzed the genome-wide binding profile of a truncated version of ARR1 (ARR1 $\triangle D D K)$. This ARR1 version does not possess the receiver domain and represents a constitutive active form of ARR 15,18 . ChIP-seq and MEME analysis of ARR1 DDDK uncovered the B-ARR-6-BA motif as the primary binding motif (Fig. 5-ARR1 $\triangle D D K+$ Dex), indicating that cytokinin can directly 
a

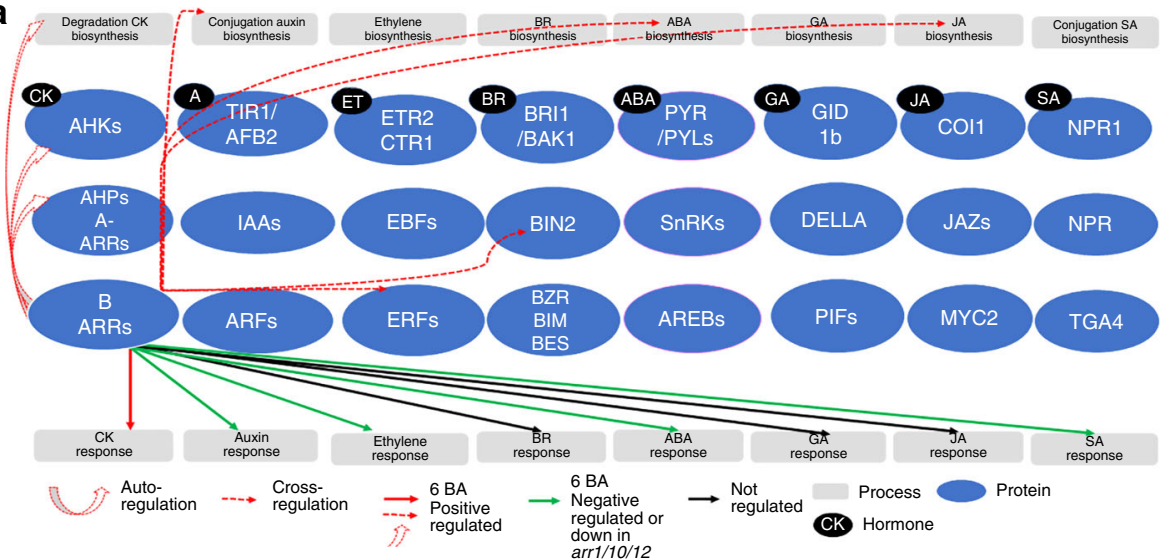

b

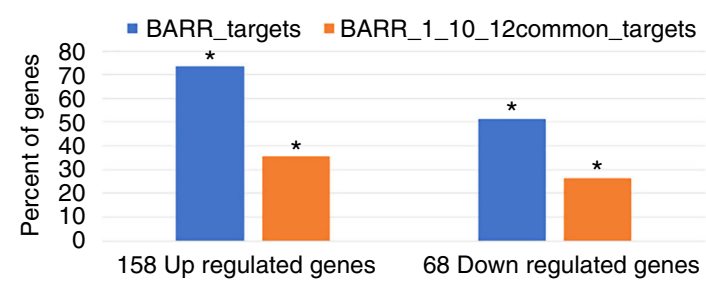

C

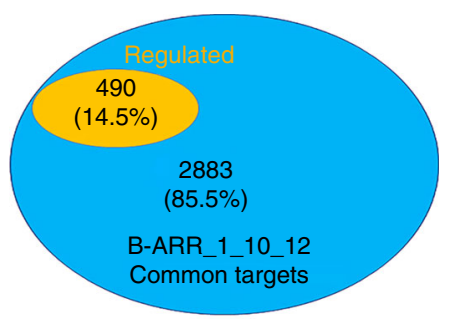

d

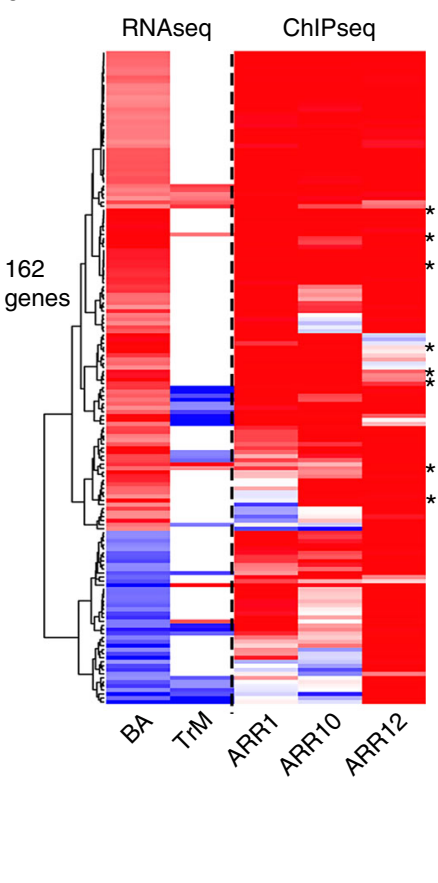

Fig. 4 Enriched GO categories for B-ARRs target genes. a Interaction graph drawn from GO categories of nearby target genes of B-ARRs. The model shows that B-ARRs regulate every step of both cytokinin biosynthesis and signaling pathways and cross-regulate other plant hormone pathways. Hormone annotation legend: CK cytokinin, A auxin, ET ethylene, BR brassinosteroid, ABA abscisic acid, GA gibberellin, JA methyl jasmonate acid, SA salicylic acid. Red arrow: genes with increased transcript levels after cytokinin treatment; Green arrow: genes with decreased transcript levels in arr1/10/12 triple mutants compared to wild type or genes repressed by cytokinin. Black arrow: genes with regulated (for BR) and not-regulated (for GA and JA) transcript levels in arr1/10/12 triple mutants compared to wild type. $\mathbf{b}$ Comparison between B-ARR targets and cytokinin response genes in the golden list ${ }^{20}$ using Fisher's exact test (asterisk $\left(^{\star}\right)$ indicates corrected $p<0.05$ ). c The subset of B-ARR 1,10 , and 12 candidate target genes also showing transcript changes (regulated). d A heatmap shows both cytokinin regulation and B-ARR binding for a subset of 162 common targets of B-ARR 1, 10, and 12. A-ARRs were highlighted $\left(^{\star}\right)$. Z scores for RNA-seq were based on log2 fold changes (6-BA treatment compared to mock and triple mutants compared to wild type) and for DNA binding were based on log2 peak score (-log2( $p$-value)). RNA-seq was acquired from 4-h 6-BA treatment (BA) or in arr1/10/12 triple mutants (TrM). DNA-binding for 6-BA treatment target genes of B-ARR-1 (ARR1), B-ARR-10 (ARR10), and B-ARR-12 (ARR12)

affect B-ARR binding through the receiver domain. Although previous in vitro-derived B-ARR binding-motifs were identified by Weirauch et al. ${ }^{53}$, our study reveals that the subfamily-1 BARRs share a similar DNA-binding motif and provides direct in vivo evidence of the DNA-binding signatures of B-ARRs. The similarity between 6-BA-treatment-specific DNA binding signature of B-ARRs and that of the constitutive active ARR1 $\triangle D D K$ provides additional insight into the in planta mechanism by which cytokinin modulates TF function. The B-ARR-6-BA motif was enriched in the promoter regions $(-1.5 \mathrm{k}$ to $+100 \mathrm{bps}$ surrounding the TSS of $83 \%$ (3334/4012) of ARR1 bound genes compared to the whole genome background (Fisher's Exact Test, $p<0.001$, Supplementary Fig. 3e). In the Zubo et al. study, $85 \%$ $(687 / 804)$ of the ARR10 "regulated target" genes also showed enrichment of the B-ARR-6-BA motif (Fisher's Exact Test, $p<$ 0.001, Supplementary Fig. 3e). While the B-ARR-6-BA motif is commonly present in $25,188(75 \%)$ of all promoters in the Arabidopsis genome, only 3334 (13\%) of these were bound by ARR1, suggesting that the motif is not sufficient to mediate the transcriptional response to cytokinin. In addition, we compared the ratio of TF binding sites identified in the $5^{\prime}$ and the $3^{\prime}$ (Supplementary Data 8 ). In the top 5\% target genes examined, there was a 2 -fold enrichment of $\left(5^{\prime} / 3^{\prime}\right)$ which dropped to 1.8 -fold in the top $10 \%$ target genes and 1.7 -fold for binding sites relative to all genes. There was also preferential binding at $5^{\prime}$ regions of the BARRs, which was consistent with the binding profile of other cytokinin regulated genes (Supplementary Data 9). These finding reveal that the top-ranking target genes have slightly preferable binding at their $5^{\prime}$ as opposed to $3^{\prime}$ but there are many ChIP-seq peaks (potential regulatory elements) present at gene $3^{\prime}$ ends as well.

B-ARRs target to WUS in stem cell maintenance. Cytokinin is a central player in shoot apical meristem initiation and maintenance 22,23 . The fact that the arr1/10/12 triple mutant produces a smaller size shoot apical meristem also implies that the cytokinin transcriptional responses are important for stem cell maintenance ${ }^{8}$. It is possible that cytokinin signaling directly targets either WUS or $C L V 3$, genes that control the meristem size, the WUS-CLV3 loop ${ }^{54,55}$. Interestingly the WUS gene which plays an important role in shoot apical meristem maintenance, a cytokinin-dependent process ${ }^{22,55}$, was a consistent low-ranking target of B-ARRs. We further explored dynamic binding of BARRs at the promoter of WUS and identified the B-ARR binding site within the promoter of WUS. After $4 \mathrm{~h}$ of cytokinin treatment, clear binding of ARR1 at the WUS promoter could be observed which became even more apparent in the 3-day hormone treatment samples (Fig. 3e). Thus, WUS is likely a, cytokinin-dependent, target of ARR1 (Fig. 3e). Similarly, cytokinininduced binding at the promoter of WUS was also found for ARR12, showing a significant increase with hormone treatment and ARR10 targeting the promoter of WUS at both mock and 
$-6-B A$

ARR1

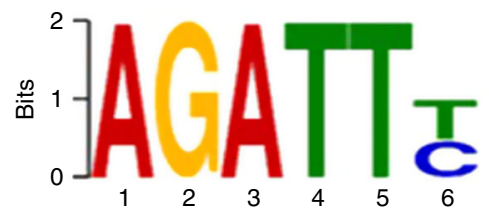

ARR10
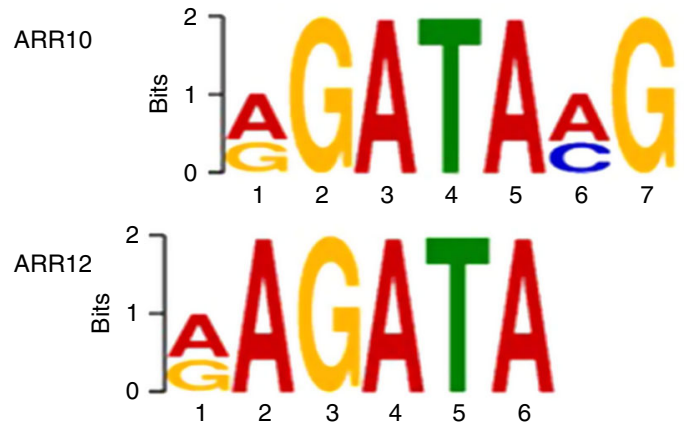

$+6-B A$
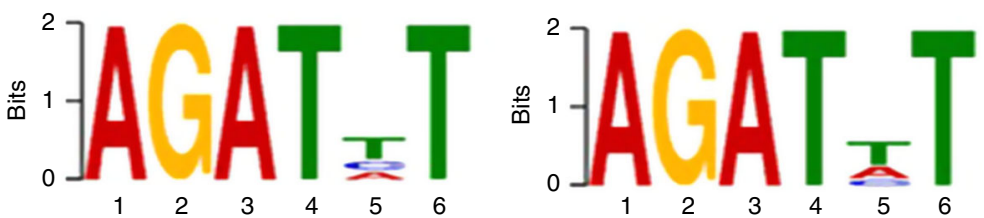
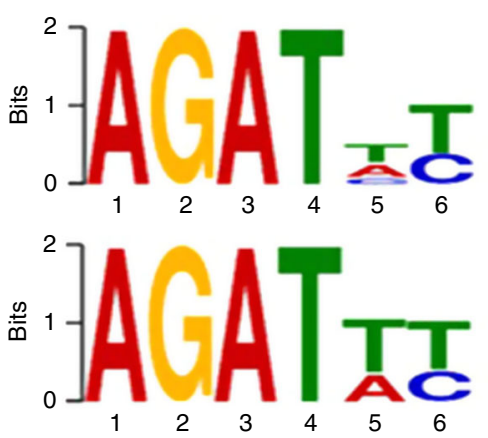

Fig. 5 Identification of the 6-BA dependent B-ARR binding motif. Similar DNA binding motifs of B-ARRs become nearly identical (B-ARR-6-BA motif) in response to increased cytokinin treatment (6-BA). AGAT is the core binding-motifs of $B-A R R s$ and $A G A T H Y, H(a / t / c)$, Y ( $t / c)$ are the $B-A R R-6-B A$ motifs after cytokinin treatment. -6-BA: DMSO, +6-BA: $10 \mu \mathrm{M}$ 6-BA. Inducible ARR1 $D D D K$ was treated using $10 \mu \mathrm{M}$ dexamethasone (ARR1 $D D D K+$ Dex)

cytokinin treatment conditions, although with less binding (Fig. 3e).

Two lines of evidence point to WUS as a candidate target of cytokinin TFs, such as the B-ARRs. First, cytokinin signaling revealed by two component sensor (TCS) is higher in WUS domain than any of the other domains in shoot apical meristem ${ }^{56}$. Second, cytokinin induces expression of a WUS transcriptional reporter gene ${ }^{22}$. Interestingly, as mentioned above WUS consistently appears as a low-ranking target gene in ChIPseq analyses for each of the tested B-ARRs in plants not exposed to exogenous cytokinin. One possibility is that binding of B-ARRs to the promoter of WUS requires an elevated level of cytokinin. When plants were grown with cytokinin, we identified a single binding site in the WUS promoter with the B-ARR-6-BA motif (AGATAT) located at the peak summit (Fig. 6a) along with increased binding (Fig. $6 \mathrm{~b}$ black bar). These findings are consistent with a previous report that activation of transcriptional reporter $p W U S: G F P e r$ in the shoot apical meristem requires high $(1 \mathrm{mM})$ concentrations of cytokinin ${ }^{22}$. Since a high concentration of cytokinin was required to activate WUS, the effect had been previously thought to be indirect. However, our results demonstrate the presence of a B-ARR-6-BA motif in the WUS promoter, implying that WUS may be an in vivo target of $\mathrm{B}$ ARRs. Interestingly, the B-ARR-6-BA motif is adjacent to HDZIP response elements, suggesting possible interactions with other TFs that may be required for stem cell maintenance in the shoot apical meristem (Fig. 6a). Indeed, recent reports provide evidence for the interaction between B-ARR and HD-ZIP ${ }^{21,57-59}$.

To understand the functional significance of B-ARRs-mediated TF binding and increased WUS gene expression, a constitutively active form of ARR1 was introduced into plants using a Dex inducible system ${ }^{60}$. Deletion of the signal receiver domain ( $\triangle D D K$ ) of $A R R 1$ (a $B-A R R$ ) has been shown to constitutively activate cytokinin signaling by unmasking the transactivation function of $\mathrm{ARR} 1^{15}$. The ARR1 deletion construct was fused to the glucocorticoid-inducible artificial transcription factor (GR) and expressed from the cauliflower mosaic virus ( $\mathrm{CaMV}$ ) $35 \mathrm{~S}$ coat protein gene promoter to generate dexamethasone (Dex) inducible 35S:ARR1 $\triangle D D K: G R^{15}$. In the absence of dexamethasone (Dex), transgenic plants carrying the 35S:ARR1 $\triangle D D K: G R$ construct were phenotypically normal (Fig. 6c, Mock). Upon dexamethasone induction, these plants displayed a phenotype similar to tissue explants propagated on cytokinin culture medium (Fig. 6c, +Dex). If B-ARR binding at the B-ARR-6-BA motif in the promoter of WUS activates WUS transcription, then, based on current knowledge of this pathway, transcriptional activation of $C L V 3$ is subsequently expected ${ }^{54,55}$. To test this model, the 35S:ARR1 $\triangle D D K: G R$ construct was introduced into plants carrying, $p C L V 3: m G F P 5-E R$ (CLV3 promoter driving the expression of endoplasmic reticulum-localized green fluorescent protein), a fluorescent reporter for stem-cells and 35S::YFP29-1 (ubiquitous promoter driving the plasma membrane-localized yellow fluorescent protein) and marker for cell boundaries which allows visualization of all SAM cells. We observed that constitutive activation of cytokinin signaling led to expansion of stem-cell domain marked by the $p C L V 3$ reporter upon induction of $A R R 1 \triangle D D K: G R$ (Fig. 6d). An alternative explanation for the activation of the $p C L V 3$ reporter would be due to increased transcription of $A-A R R s$ by the $35 S: A R R 1 \triangle D D K$ as a previous study reported the requirement of $A R R 7$ and $A R R 15$ to maintain $C L V 3$ expression $^{23}$. However, the size of shoot apical meristem in 35S:ARR1 $\triangle D D K: G R$ system did not increase. We then tested the effect of expression of $A R R 1 \triangle D D K$ under control of the $p C L V 3$ promoter using $p C L V 3: L h G 4 / p M X 6 x O P s: A R R 1 \triangle D D K: G R$, a twocomponent inducible system. An enlarged shoot apical meristem ("ball" of SAM) was observed. When expression of the transgene was induced by Dex treatment, all ten characterized lines harboring both $p C L V 3: L h G 4$ and $p M X 6 \times O P s: A R R 1 \triangle D D K: G R$ showed similar phenotypes at the seedling stage (Fig. 6e, +Dex 12 days and + Dex 19 days). Plants were first grown on MS plates and then transferred to $10 \mu \mathrm{M}$ Dex plate for induction, phenotypes observed in adult transgenic plants included a "ball" of the primary SAM (Supplementary Fig. 7a), indeterminate floral meristem (Supplementary Fig. 7a), and a "ball" of the lateral SAM (Supplementary Fig. 7a, "Ball" of Lateral SAM). The observed "ball" of the SAM phenotype is reminiscent of the "dome" shape of SAM in transgenic plants observed when WUS is expressed under the control of the $p C L V 3$ promoter ${ }^{61}$. The expression of WUS in $p C L V 3:$ LhG4/pMX6xOPs inducible system was also reported to have an enlarged shoot apical meristem ${ }^{62}$. Transgenic plants germinated on MS plates and then transferred to $10 \mu \mathrm{M}$ Dex plates, both $p C L V 3: L h G 4 / p M X 6 x O P s: W U S: G R$ and $p C L V 3$ : 
a

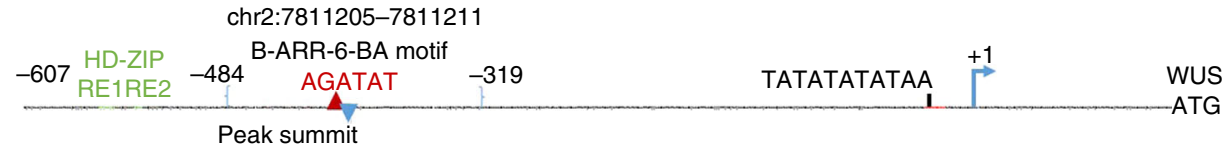

b

(chr 2: 7811185-7811186)

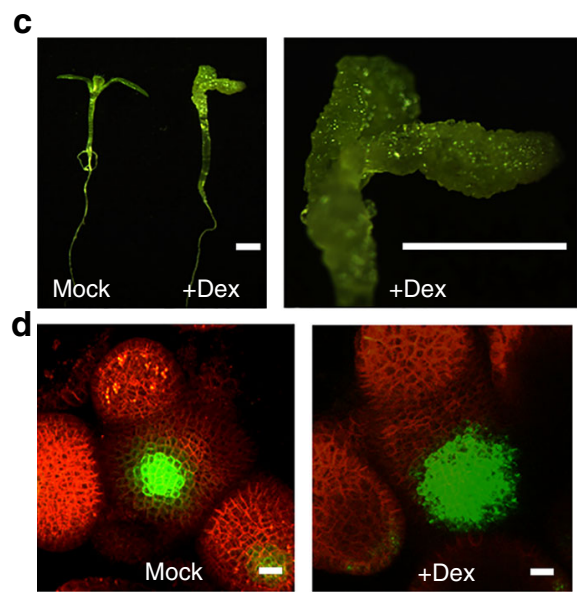

e
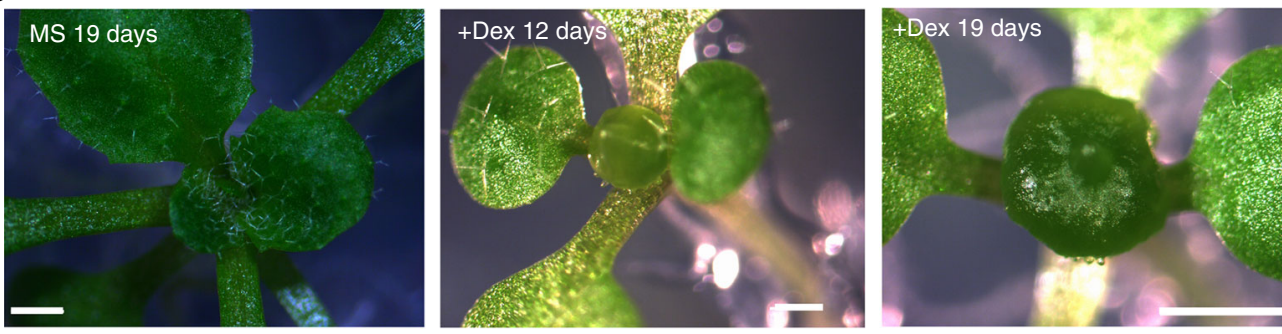

f
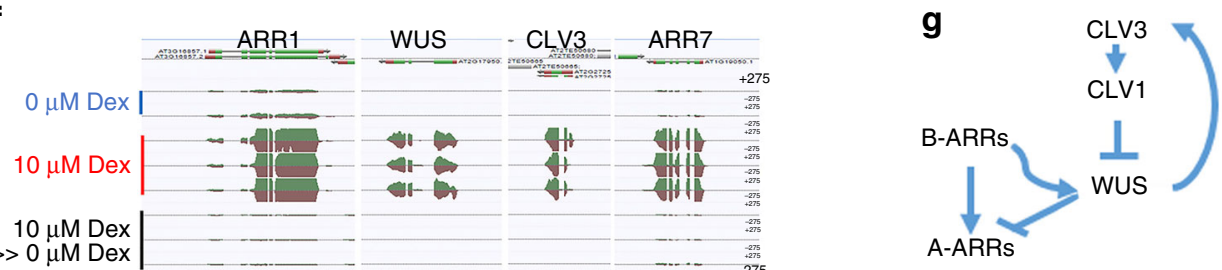

Fig. 6 Cytokinin dependent B-ARRs targeting of the WUS promoter. a The location of the AGATAT B-ARR-6-BA motif relative to the WUS gene's TSS. The number (7811185) of the peak summit is the genomic position on the second chromosome. $\mathbf{b}$ An AnnoJ snapshot showing B-ARRs binding to WUS promoter requires a high concentration of cytokinin. $Y$-axis heights are scaled to same value. Black bar indicates plants grown on plates with $10 \mu \mathrm{M}$ 6-BA for 3 days (BA3d) or ARR1ADDK:::GR plants treated with $10 \mu \mathrm{M}$ Dex. c, d Phenotypes of p35S::ARR1ADDK::GR. c Phenotypes of 11 days old transgenic plants without Dex (Mock), with Dex (+Dex), and at higher magnification (+Dex). Scale bar $=1 \mathrm{~mm}$. d 3D-reconstructed views of shoot apical meristems (SAMs) showing pCLV3::mGFP5-ER (CZ/stem cell reporter; green) and 35S::YFP29-1 (plasma membrane-localized YFP marks the outlines of all cells, red). SAM of mock-treated plants 4 days after treatment (Mock) and SAM of Dex-treated plants 4 days after treatment ( + Dex). Scale bar $=10 \mu M$. e Enlarged shoot apical meristem ("ball" of SAM)) by the inducible expression of the constitutive active form ARR1 (ARR1 $D D D K: G R$ ) in stem cell domain (CLV3 domain). Scale bar $=1 \mathrm{~mm}$. The transgenic plants without Dex induction as a control after growth on MS for 19 days (MS 19 days). "Ball" of SAM after growth on $10 \mu \mathrm{M}$ Dex plate for 12 days (+Dex 12 days). "Ball" of SAM after growth on $10 \mu \mathrm{M}$ Dex plate for 19 days (+Dex 19 days). f RNA-seq reads tracks of the "ball" of SAM system demonstrating the expression of ARR1, ARR7, WUS, and CLV3. Transgenic plants grown on MS for 19 days (0 $\mu M$ Dex), on 10 $\mu \mathrm{M}$ Dex plate for 19 days (10 $\mu \mathrm{M}$ Dex) and grown on $10 \mu \mathrm{M}$ Dex plate for 12 days then transferred to MS for 7 days (10 $\mu \mathrm{M}$ Dex $>0 \mu \mathrm{M}$ Dex). All tracks have the same scaling of $y$-axes (+275/-275). $\mathbf{g}$ A sketch showing the interface between the cytokinin response and the CLV3-WUS circuit

LhG4/PMX6xOPs:ARR1 $\triangle D D K: G R$ also showed a similar "ball" of SAM phenotype (Supplementary Fig. 7b). RNA-seq of "ball" cells isolated from the SAM clearly showed ARRIDDDK was overexpressed compared to the full length of ARR1 (Fig. 6f). Moreover, transcriptional activation of both WUS, and CLV3 was observed (Fig. 6f) although CLV3 was not found to be a target of any of the tested B-ARRs. These results are consistent with the hypothesis that B-ARRs activate WUS expression, which in turn activates CLV3. Therefore, our results provided a link between the cytokinin transcriptional response and the WUS-CLV3 circuit in the shoot apical meristem (Fig. $6 \mathrm{~g}$ ).

\section{Discussion}

In this study, we employed recombineering to engineer a Ypet-tag onto three B-ARRs, ARR1, ARR10, and ARR12. This system enabled monitoring of endogenous expression and protein localization patterns for three B-ARR TFs. It also allows real time 
visualization of the cytokinin primary TFs which was previously only done by a GUS fusion. The recombineered B-ARRs were also used for ChIP-seq experiments, allowing the identification of in vivo binding sites and putative target genes. Comparison of binding profiles from both endogenous and elevated cytokinin conditions showed marked differences in both motif sequence and target gene number. Based on these dosage experiments, there may be as many as 10,000 cytokinin response genes in the $B-A R R$ network. Thus, a limiting factor might be the amount of endogenous cytokinin to modify B-ARRs.

The genome-wide identification of the targets of B-ARRs provides a new resource to understand how cytokinin may regulate diverse plant growth and developmental processes, as well as respond to stresses in conjunction with other phytohormones at different regulatory layers, such as biosynthesis, transportation, perception, or signal transduction. The tissue/cell expression patterns of ARR1, ARR10, and ARR12 are quite similar ${ }^{29}$. Similarly, in vivo ChIP-seq targets for the three B-ARRs also resemble one another. When cytokinin levels are elevated, B-ARRs target many plant hormone negative regulators, such as $A-A R R s^{63}, A u x /$ $I A A s^{64}$, and $E B F s^{47}$. Targeting of multiple negative regulators in multiple hormone pathways might provide a quick and effective avenue to abate this hormone imbalance ensuring quick reequilibration of responses. Additionally, a recent ChIP-seq study using tagged, over-expressed ARR10 identified a set of ARR10bound cytokinin responsive genes ${ }^{21}$. Since over-expression of $A R R 10$ was able to rescue arr1/10/12 triple mutants ${ }^{21}$, it is not surprising that the list of targets $(81.5 \%$ or 3265 out of 4004 genes) shows a statistically significant overlap with the targets that we identified using recombineered ARR genes (Supplementary Fig. 3b, Fisher's exact test, $p<0.001)$. The ARR10 overexpressing ChIP-Seq results shared 2783 gene targets with our ARR10_BA and had 1221 unique targets not identified by ARR10_BA. Interestingly, the 2783 overlapping target genes found by both studies had higher peak scores than unique target genes (Supplementary Fig. 8a), suggesting that these are high confidence ARR target genes. However, expression of the native level of the ARRs using recombineered genes may allow the identification of more authentic, cytokinin-response relevant target genes. In this regard, dataset 1 of Zubo et al. contained 1221 additional targets that did not show enrichment for any meaningful GO terms while 3489 targets unique to the recombineering ARR10 ChIP-seq data showed significant enrichment for plant hormone GO terms (Supplementary Fig. 3b). Moreover, over-expression of ARR10 identified known target gene for other B-ARRs (Supplementary Fig. 3b and 3d; Fig. 8b). However, the number of unique targets dropped to 739 when we compared these binding sites to ChIP-seq results for other B-ARR 6-BA treated tissues (Supplementary Fig. 3b, 3d). Moreover, the 482 targets shared between other recombineered B-ARRs and ARR10 over-expressing line had lower peak scores compared to the 2783 shared ARR10 target genes between our study and Zubo et al. (Supplementary Fig. 8b), suggesting potential off-target binding in ChIP-seq experiments using ARR10 over-expressing plants. Combined, these studies provide biochemical evidence confirming genetic redundancy among B-ARR factors (Supplementary Fig. 3d). Interestingly we also uncovered a change of the B-ARR binding motif upon cytokinin treatment which was likely missed in earlier studies since this observation requires comparing B-ARRs at both endogenous conditions and cytokinin treatment (Fig. 5). Finally, gene targets identified by over-expression of ARR10 by Zubo et $\mathrm{al}^{21}$. identified a number of the top-ranking B-ARR targets in our study (Supplementary Data 10). Thus, our findings provide unique information not available in previous studies, providing novel insight about full response of the plants to cytokinin.
Although several types of in vitro experiments identified potential DNA binding motifs for B-ARRs, the in vivo binding sites identified here provided a unique opportunity to further analyze DNA binding events. Cytokinin promoted B-ARR motif switching, from a more degenerated motif to a canonical B-ARR6-BA motif (AGATHY). B-ARRs are not regulated at transcriptional level by cytokinin but are post-transcriptionally modified $^{11,13}$. One possibility is that without such modification BARRs only loosely bind their targets; further studies are necessary to explore the impact of phosphorylation on the motif site selection. ARR1 showed the highest cytokinin-dependent enrichment of binding to its targets. Without cytokinin treatment, only 2815 potential targets were identified but the number increased to 5128 after just four hours of cytokinin treatment, and it further increased to 10,340 targets when treated for 3 days. These targets could be further arranged in a hierarchical manner into early binding (targets found in both mock and 4-hour BA treatment as 1st), short BA treatment binding (targets found in both 4-hour and 3-day BA treatment as 2nd), and longer BA treatment binding (targets found only in 3-day BA treatment as $3^{\text {rd }}$ ) (Supplementary Fig. 3f). We speculate that longer cytokinin treatment may change chromatin structures such that more binding sites become available. Although the high dose and 3-day treatment may potentially result in false positives peaks, the high overlap between lower level hormone treatments and these highdose experiments confirm the relevant cytokinin responsiveness. This cytokinin-dependent binding of ARR1 to its targets might be explained by hormone-dependent alteration in ARR1 protein stability. Alternatively, a high concentration of cytokinin may trigger the phosphorylation cascade resulting in activation of TF B-ARRs, and these phosphorylated B-ARRs may bind their targets more tightly than those lacking the modification. In this twocomponent multiple phosphorelay system, it is thought that phospho-activated B-ARRs change the conformation of their receiver domain ${ }^{15,18}$. The model asserts that B-ARRs constitutively occupy their binding sites, only becoming "active" upon phosphorylation ${ }^{65}$. Our time-series ChIP-seq analysis provides correlative evidence that cytokinin increases both binding of B-ARRs to their targets and the number of targets bound. However, this suggestion must be further addressed by complementary experiments, such as the reduction of endogenous cytokinin levels using a regulated expression of $C K X 3^{66}$ to examine the impact of endogenous cytokinin removal on the interaction between B-ARRs and their targeted promoters. Alternatively, using mutations at the conserved phosphorylation sites in B-ARRs may also help to test this model ${ }^{67}$.

Importantly, we identified a B-ARR binding site in the promoter of WUS gene, which encodes a homeodomain TF that was shown to repress a subset of A-ARRs ${ }^{24,55}$. WUS has been suggested to work in conjunction with the cytokinin pathway ${ }^{22,24}$ to establish a stem cell niche ensuring early embryogenesis and later for maintenance of the shoot apical meristem ${ }^{55}$. The study using the new reporter revealed that the WUS expression domain overlaps with SAM regions where cytokinin activity is the highest ${ }^{68}$. Similarly, the $p W U S:: G F P$-er reporter can be activated in SAM by cytokinin but only at a high concentration of cytokinin. However, subsequent (expected) activation of a $p C L V 3:: G F P$-er reporter was not observed ${ }^{22}$. A previous explanation of why WUS does not robustly respond to low cytokinin levels was that the expression of WUS was only in the a specific (WUS) subdomain of the SAM and that the cytokinin was not accessible to the WUS domain. Our results indicate that most of the potential binding motifs of B-ARRs in the promoter region of WUS were unoccupied. However, we identified one B-ARR-6-BA motif, located precisely at the peak summit of the conservative narrow peak, in multiple B-ARRs data sets. The identification of a strong in vivo 
B-ARR DNA binding site within the promoter of WUS that requires a high level of cytokinin, provides new direct evidence for this association. In addition, the B-ARR-6-BA motif (-420bp) was found to be within $57 \mathrm{bps}$ as two HD ZIP response elements $(-540$ to -565$)$ that were previously identified as WUS regulatory sites ${ }^{69}$. It is possible that the coordination or competition among these TFs is a feature of WUS regulation, which is itself controlled by a WUS-CLV3 negative feedback loop, keeping the meristem size constant in each species. Moreover, the activation of WUS by B-ARRs downstream of cytokinin signaling should also be tightly attenuated in the shoot apical meristem. When a constitutive active form of $A R R 1$ was introduced, removing the possibility of feedback inhibition, the activation of WUS led to an expansion of stem cell domain. Interestingly, ectopic expression of $A R R 1 \triangle D D K$ did not lead to an enlarged SAM, indicating the possible presence of an unknown inhibition mechanism from other domains within the SAM. Expression of ARR $\triangle D D D$, under the control of $p C L V 3$ promoter, resulted in an enlarged shoot apical meristem ("ball" of SAM). This phenotype may result from activation of WUS by B-ARRs in the stem cell that cannot be dampened. Since type-A ARRs were highly activated by B-ARRs, increasing the level of type-A ARRs may cause increased expression of $C L V 3$ as $A R R 7$ and $A R R 15$ are required for the expression of $C L V 3^{23}$. However, as negative regulators, the inhibition mechanism of type-A ARRs to cytokinin signaling remains unknown ${ }^{11}$. Similarly, the activation mechanism of type-A ARRs leading to $C L V 3$ expression is also not clear. If $C L V 3$ is the solely interface between B-ARR and A-ARRs leading to activate of $C L V 3$, then transcription of WUS would be expected to shut down.

The "ball" of the SAM phenotype and high levels of both WUS and CLV3 expression observed in pCLV3:ARR1 $D D D K$ system suggests that B-ARRs activate the transcription of WUS, then activate CLV3 leading to expand the shoot apical meristem, resulting in the "ball "of SAM. Previous reports of $p C L V 3: W U S$ expression resulted in similar phenotypes, further providing functional relevance to our model ${ }^{61,62}$. This result is also consistent with the idea that multiple feedback loops exist in the shoot apical meristem to adjust its size ${ }^{22}$. The introduction of a mutation in the B-ARR binding site in the promoter of WUS would test the idea that WUS is, at least in part, under direct transcriptional control of B-ARRs. Taken together, these findings provide new insights to the role of the cytokinin transcriptional responses in stem cell maintenance.

\section{Methods \\ Plant growth conditions. Three-days-old seedling tissue was collected for these experiments unless otherwise noted. Seeds were surface-sterilized and sown on agar plates (1.8\%) containing Murashige and Skoog salts ( $\mathrm{pH}$ 5.7) and 1\% sucrose. Seedlings were grown vertically for 3 days at $22^{\circ} \mathrm{C}$ under long day condition using a 16-h light/8-h dark cycle. The seedling was subsequently treated with liquid Murashige and Skoog medium (MS) pH 5.7 with $0.08 \%$ Silwet 77 containing $10 \mu \mathrm{M}$ 6-BA (in DMSO) or mock treated with an equivalent volume of DMSO. The three- days continuous hormone treatment was orchestrated by germinating the tagged lines on MS with $10 \mu \mathrm{M}$ 6-BA or MS with the same amount of DMSO as mock. 10 $\mu \mathrm{M}$ 6-BA was chosen based on the range from $1 \mu \mathrm{M}$ to $20 \mu \mathrm{M}$ cytokinin treatment ${ }^{20}$}

Gene constructs and generation of tagged B-ARRs. Based on recombineering techniques ${ }^{28}$, a Ypet (yellow fluorescent protein) tag was recombineered into a transformable bacteria clone (TAC) clone such that the B-ARR gene was located at the center of the large insert clone. We employed the two-step recombineering method using the Flapase-Fret system. Positive Ypet clones were obtained first by selection against ampicillin as the insert contained an ampicillin resistant gene. This ampicillin marker was later removed. While the technique leaves a small scar between Ypet and the B-ARRs the method is easier than the GalK system ${ }^{28}$ and the scar serve as a linker between the tag and the B-ARRs. The Ypet gene was added at the C-terminus of each B-ARR gene of the whole B-ARR family but only ARRs 1, $10,12,11,13$, and 14 were successful. ARR1 was tagged using isoform

ARR1.2 since the previous expression data indicated ARR1.2 as the major splice variant. The tagged TACs were sequenced to confirm sequence fidelity of the BARRs, the junction, and the fusion to Ypet within TAC. The tagged TAC clones were transformed into GV3101 strains and transformed wild type Columbia-0 plants using the flower dipping method ${ }^{70}$. After screening for Basta resistance, putative tagged lines were identified by PCR using forward recombineering test primer and reverse Ypet primers. The PCR products were gel-purified and sequenced to confirm the in planta tagging junction. The expression of the tagged B-ARRs (ARR1, ARR10, and ARR12) was then examined under Zeiss confocal microscopy 710 (Zeiss) using identical settings (6\% laser power; master gain: ch1 910, ch2 248; digital gain 1, digital offset 0; pinhole $281 \mu \mathrm{M}$; filter ch1 521-546).

The active form of ARR1 was constructed using a deletion of the receiver domain of ARR1 that was previously described ${ }^{15,18}$. The deletion called $A R R 1 \triangle D D K$ was cloned into $p E N T R D / T O P O$ with GR fusion at the C-terminus and then the entire $A R R 1 \triangle D D K: G R$ gene cassette was moved into the $p E G 104$ transformation vector to be in frame of a N-terminus $Y F P^{71}$. The $p E N T R$ D/TOPO: $A R R 1 \triangle D D K: G R$ was cloned into $p M X 6 x O P s$ vector and then the transgenic line was combined with the $p C L V 3: L h G 4$ line to achieve stem cell-specific expression of ARR1 $\triangle D D K$

Observation of phenotypes using the inducible system. The two-components inducible system was used ${ }^{62}$. The $p C L V 3: L h G 4$ line was in Landsberg erecta (Ler- 0$)$ background. The $p M X 6 \times O P s: A R R 1 \triangle D D K: G R$ was transformed into Ler-0 back ground. The $p M X 6 x O P s: A R R 1 \triangle D D K: G R$ lines were screened using gentamicin. Ten individual lines were used to cross with the $p C L V 3: L h G 4$ line and were tested on MS medium with $10 \mu \mathrm{M}$ Dexamethasone (Dex). MS medium with the same amount of ethanol was used as control. All ten lines showed the enlarged apical meristem phenotype. Initial observation of phenotypes was done by germinating seeds directly on Dex plates. The pCLV3:LhG4/pMX6xOPs:WUS:GR line was provided by Dr. Reddy's lab and the same induction method was used.

Chromatin preparation and immunoprecipitation. Seedlings were dried by paper towel and transferred in 1\% formaldehyde solution. Cross-linking occurred under 5-10-5-min vacuum cycles with a quick vacuum release in between each cycle. A final concentration of $125 \mathrm{mM}$ glycine was applied for $5 \mathrm{~min}$ to deactivate the remaining formaldehyde. Cross-linking resulted in translucent seedling tissue. Tissue was then liquid nitrogen cooled and either stored in $-80^{\circ} \mathrm{C}$ degree or directly ground and an extraction of chromatin was performed as previously described $^{36}$. Chromatin immunoprecipitation (ChIP) was performed as previously described $^{36}$ with modifications, including the use of Bioruptor sonicator (Diagenode, Belgium). Bioruptor settings used were: Low, 10 cycles of $25 \mathrm{~s}$ on, $120 \mathrm{~s}$ off. Sonication was performed in auto-cooling system with water bath at $4{ }^{\circ} \mathrm{C}$. A small amount of chromatin $(10 \mu \mathrm{l})$ was evaluated for shearing; the size range of chromatin was $150-450$ bps, most fragments at 250 bps.

The commercial anti-GFP antibody (Thermo Fisher Scientific, A11111) was used for the immunoprecipitation reactions. Five microgram antibody and $50 \mu \mathrm{l}$ of Dynabeads M-280 Sheep anti-Rabbit IgG (Thermo Fisher Scientific, catalog \#11204D) were consumed for each reaction. The incubation was performed using previously described buffer ${ }^{36}$ at $4{ }^{\circ} \mathrm{C}$ overnight $(16 \mathrm{~h})$. The Dynabeads were washed using a low stringent wash buffer followed by a high stringent buffer $^{36}$, a quick rinsing, and $5 \mathrm{~min}$ rotating at $4^{\circ} \mathrm{C}$. A final wash buffer was applied to clear the detergent from previous buffers followed by another 5 min rotating. During each wash, the tubes were quickly centrifuged before returning to the magnetic stand and trace amounts of buffer were removed to avoid non-specific binding carryover. The resulting ChIP DNA was collected in two elution buffers $(100 \mu \mathrm{l} \mathrm{each})$ at $65^{\circ} \mathrm{C}$ and combined. The proteinase $\mathrm{K}$ digestion occurred at $55^{\circ} \mathrm{C}$. The reverse of crosslinking was done at $65^{\circ} \mathrm{C}$ overnight $(16 \mathrm{~h})$. The ChIP DNA was then purified by extraction using phenol:chloroform:isoamyl alcohol (25:24:1) (Sigma, p3803) twice in a phase lock gel system before the ethanol precipitation step using glycogen for the pellet observation. The pellets were then washed with $70 \%$ cooled ethanol and dried in a speed-vac. The ChIP DNA was then dissolved in $50 \mu \mathrm{l}$ water for subsequent library preparation.

ChIP-seq library generation and sequencing. The single-end read libraries were prepared using the TruSeq ChIP kit from Illumina (catalog ID: IP-202-1024), with DNA size selection made with lab-made Serapure magnetic beads. Library concentration were assayed using the Qubit 2.0 Fluorometer (Thermo Fisher Scientific) and normalized to 1.7 Nano gram per microliter. Six multiplexed libraries were sequenced per lane on a HiSeq 2000.

ChIP-seq data analysis. ChIP-seq data analyses were carried out using a suite of pipelines developed in our lab to run the alignment and analysis. Briefly, alignment was performed using Bowtiel with parameters " -m 1-best-strata -S-chunkmbs $200^{\prime \prime 2}$. MACS2 was used to call peaks compared to input using q_value_thresholds $=0.05,0.01,0.001$. The aligned reads with at least two biological replicates were processed using the irreproducibility discovery rate (IDR) ${ }^{73}$. The $p$-value cutoff was 1e-16. Peaks were then annotated using ChIPpeakAnno package. In order for us to classify a peak as associating with a gene, the peak summit must have been within $1.5 \mathrm{~kb}$ upstream and downstream of the gene's annotation. The gene length was normalized to $1 \mathrm{~kb}$ to describe average enrichment of peaks around 
gene body TSS. The B-ARR-6-BA motif matrix was used by $\mathrm{Homer}^{74}$ to identify the locations of the motif sequence on the whole genome. Promoter regions were defined as -1500 to +100 bps relative to the TSS. Gene ontology analysis was conducted using DAVID GO ${ }^{37 \text {; }}$ the top 3000 genes ranked by IDR peak score of each experiment were evaluated for GO term overrepresentation (The $p$-values were corrected for multiple testing). Total 200 bps flanking the binding summits of 1000 top-ranking peaks for each experiment were also used for identification of each DNA binding motif using MEME-ChIP suite ${ }^{75}$. The list of genes associated with cytokinin pathway was downloaded from Gene Ontology Consortium (http:// www.geneontology.org $)^{76}$. The heatmap of the B-ARRs' target genes was hierarchical clustered based on the Euclidean distances, calculated from the number of shared targets between each factor for different conditions. A directed network was constructed based on the relationship between TFs and their binding targets by igraph (http://igraph.org) ${ }^{77}$ with nodes representing either type-A ARR or TFs with significant transcriptional changes defined by the meta-analysis (Supplementary Data 5) and edges representing either ChIP-Seq binding of type-B ARR or DAP$\mathrm{Seq}^{33}$ binding of TFs. The network was visualized in Cytoscape $(\mathrm{v} 3 \cdot 4 \cdot)^{78}$. DAP-Seq data were downloaded from the website (http://neomorph.salk.edu/dap_web/ pages/index.php) and only samples without "amp" label were used for this analysis $^{33}$

RNA-seq and data analysis. To compare the arr1/10/12 triple mutant with the wild type, three-days-old seedlings were collected without any treatment. For cytokinin treatment, $10 \mu \mathrm{M}$ 6-BA or an equal volume of ethanol was applied to each sample. RNA was isolated using RNeasy plant kit (Qiagen, cat \#74904) and libraries were prepared by NeoPre Library Prep System (Illumina). Alignments were done by Tophat 2(v2.0.8, using TAIR10, Bowtie 2, and default parameters) ${ }^{79}$ and differential expression was called by CuffDiff (Cufflinks v2.1.1, using TAIR10 and default parameters $)^{80}$. The significantly differentially expressed genes used 1.6fold change and $q$-value $<=0.05$ as cutoff.

Data availability. Raw and processed data can be found with GEO deposition accession number GSE94486. The authors declare that all other data supporting the findings of this study are available within the manuscript and its supplementary files or are available from the corresponding author upon request.

Received: 8 June 2017 Accepted: 22 March 2018

Published online: 23 April 2018

\section{References}

1. Tran, L. S. et al. Functional analysis of AHK1/ATHK1 and cytokinin receptor histidine kinases in response to abscisic acid, drought, and salt stress in Arabidopsis. Proc. Natl Acad. Sci. USA 104, 20623-20628 (2007).

2. Argyros, R. D. et al. Type B response regulators of Arabidopsis play key roles in cytokinin signaling and plant development. Plant Cell 20, 2102-2116 (2008).

3. Werner, T. \& Schmülling, T. Cytokinin action in plant development. Curr. Opin. Plant Biol. 12, 527-538 (2009).

4. Ha, S., Vankova, R., Yamaguchi-Shinozaki, K., Shinozaki, K. \& Tran, L. S. Cytokinins: metabolism and function in plant adaptation to environmental stresses. Trends Plant Sci. 17, 172-179 (2012).

5. Schaller, G. E., Bishopp, A. \& Kieber, J. J. The yin-yang of hormones: cytokinin and auxin interactions in plant development. Plant Cell 27, 44-63 (2015).

6. Skoog, F. \& Miller, C. O. Chemical regulation of growth and organ formation in plant tissues cultured in vitro. Symp. Soc. Exp. Biol. 54, 118-130 (1957).

7. Higuchi, M. et al. In planta functions of the Arabidopsis cytokinin receptor family. Proc. Natl Acad. Sci. USA 101, 8821-8826 (2004).

8. Mason, M. G. et al. Multiple type-B response regulators mediate cytokinin signal transduction in Arabidopsis. Plant Cell 17, 3007-3018 (2005).

9. Hutchison, C. E. et al. The Arabidopsis histidine phosphotransfer proteins are redundant positive regulators of cytokinin signaling. Plant Cell 18, 3073-3087 (2006).

10. Yokoyama, A. et al. Type-B ARR transcription factors, ARR10 and ARR12, are implicated in cytokinin-mediated regulation of protoxylem differentiation in roots of Arabidopsis thaliana. Plant Cell Physiol. 48, 84-96 (2007).

11. Kieber, J. J. \& Schaller, G. E. Cytokinins. The Arabidopsis Book 12, e0168 (2014).

12. To, J. P. et al. Type-A Arabidopsis response regulators are partially redundant negative regulators of cytokinin signaling. Plant Cell 16, 658-671 (2004).

13. Schaller, G. E., Kieber, J. J. \& Shiu, S. H. Two-component signaling elements and histidyl-aspartyl phosphorelays. The Arabidopsis Book 6, e0112 (2008).

14. Inoue, $\mathrm{T}$. et al. Identification of CRE1 as a cytokinin receptor from Arabidopsis. Nature 409, 1060-1063 (2001).

15. Sakai, H. et al. ARR1, a transcription factor for genes immediately responsive to cytokinins. Science 294, 1519-1521 (2001).
16. Besnard, F., Rozier, F. \& Vernoux, T. The AHP6 cytokinin signaling inhibitor mediates an auxin-cytokinin crosstalk that regulates the timing of organ initiation at the shoot apical meristem. Plant Signal. Behav. 9, e28788 (2014).

17. Kim, H. J., Chiang, Y. H., Kieber, J. J. \& Schaller, G. E. SCF(KMD) controls cytokinin signaling by regulating the degradation of type-B response regulators. Proc. Natl Acad. Sci. USA 110, 10028-10033 (2013).

18. Sakai, H., Aoyama, T. \& Oka, A. Arabidopsis ARR1 and ARR2 response regulators operate as transcriptional activators. Plant. J. 24, 703-711 (2000).

19. Taniguchi, M., Sasaki, N., Tsuge, T., Aoyama, T. \& Oka, A. ARR1 directly activates cytokinin response genes that encode proteins with diverse regulatory functions. Plant Cell Physiol. 48, 263-277 (2007).

20. Bhargava, A. et al. Identification of cytokinin-responsive genes using microarray meta-analysis and RNA-Seq in Arabidopsis. Plant Physiol. 162, 272-294 (2013).

21. Zubo, Y. O. et al. Cytokinin induces genome-wide binding of the type-B response regulator ARR10 to regulate growth and development in Arabidopsis. Proc. Natl Acad. Sci. USA 114, E5995-E6004 (2017).

22. Gordon, S. P., Chickarmane, V. S., Ohno, C. \& Meyerowitz, E. M. Multiple feedback loops through cytokinin signaling control stem cell number within the Arabidopsis shoot meristem. Proc. Natl Acad. Sci. USA 106, 16529-16534 (2009).

23. Zhao, Z. et al. Hormonal control of the shoot stem-cell niche. Nature $\mathbf{4 6 5}$, 1089-1092 (2010).

24. Leibfried, A. et al. WUSCHEL controls meristem function by direct regulation of cytokinin-inducible response regulators. Nature 438, 1172-1175 (2005).

25. Tokunaga, H. et al. Arabidopsis lonely guy (LOG) multiple mutants reveal a central role of the LOG-dependent pathway in cytokinin activation. Plant $J$. 69, 355-365 (2012).

26. Schmulling, T., Werner, T., Riefler, M. \& Krupkova, E. \& y Manns, IB. Structure and function of cytokinin oxidase/dehydrogenase genes of maize, rice, Arabidopsis and other species. J. Plant Res. 116, 241-252 (2003).

27. Jones, M. G., Fish, N. \& Lindsey, K. Plant tissue culture. Methods Mol. Biol. 4, 499-517 (1988)

28. Zhou, R., Benavente, L. M., Stepanova, A. N. \& Alonso, J. M. A recombineering-based gene tagging system for Arabidopsis. Plant J. 66, 712-723 (2011)

29. Mason, M. G., Li, J., Mathews, D. E., Kieber, J. J. \& Schaller, G. E. Type-B response regulators display overlapping expression patterns in Arabidopsis. Plant Physiol. 135, 927-937 (2004).

30. Hosoda, K. et al. Molecular structure of the GARP family of plant Myb-related DNA binding motifs of the Arabidopsis response regulators. Plant Cell 14, 2015-2029 (2002)

31. Imamura, A., Yoshino, Y. \& Mizuno, T. Cellular localization of the signaling components of Arabidopsis His-to-Asp phosphorelay. Biosci. Biotechnol. Biochem. 65, 2113-2117 (2001).

32. Mason, M. G. et al. Type-B response regulators ARR1 and ARR12 regulate expression of AtHKT1;1 and accumulation of sodium in Arabidopsis shoots. Plant J. 64, 753-763 (2010).

33. O'Malley, R. C. et al. Cistrome and epicistrome features shape the regulatory DNA landscape. Cell 165, 1280-1292 (2016).

34. Brandstatter, I. \& Kieber, J. J. Two genes with similarity to bacterial response regulators are rapidly and specifically induced by cytokinin in Arabidopsis. Plant Cell 10, 1009-1019 (1998)

35. Ramireddy, E., Brenner, W. G., Pfeifer, A., Heyl, A. \& Schmülling, T. In planta analysis of a cis-regulatory cytokinin response motif in Arabidopsis and identification of a novel enhancer sequence. Plant Cell Physiol. 54, 1079-1092 (2013).

36. Chang, K. N. et al. Temporal transcriptional response to ethylene gas drives growth hormone cross-regulation in Arabidopsis. eLife 2, e00675 (2013).

37. Huang, D. W. et al. The DAVID gene functional classification tool: a novel biological module-centric algorithm to functionally analyze large gene lists. Genome Biol. 8, R183 (2007).

38. Gfeller, A., Liechti, R. \& Farmer, E. E. Arabidopsis jasmonate signaling pathway. Sci. Signal. 3, cm4 (2010).

39. Pfeiffer, A., Shi, H., Tepperman, J. M., Zhang, Y. \& Quail, P. H. Combinatorial complexity in a transcriptionally centered signaling hub in Arabidopsis. Mol. Plant 7, 1598-1618 (2014).

40. Song, Y. et al. Age-triggered and dark-induced leaf senescence require the bHLH transcription factors PIF3, 4, and 5. Mol. Plant 7, 1776-1787 (2014).

41. Zhang, Y. et al. A quartet of PIF bHLH factors provides a transcriptionally centered signaling hub that regulates seedling morphogenesis through differential expression-patterning of shared target genes in Arabidopsis. PLoS Genet. 9, e1003244 (2013).

42. Müller, M. \& Munné-Bosch, S. Ethylene response factors: a key regulatory hub in hormone and stress signaling. Plant Physiol. 169, 32-41 (2015).

43. Wang, Z. Y. et al. The brassinosteroid signal transduction pathway. Cell Res. 16, 427-434 (2006). 
44. Park, S. Y. et al. Abscisic acid inhibits type $2 \mathrm{C}$ protein phosphatases via the PYR/PYL family of START proteins. Science 324, 1068-1071 (2009).

45. Strader, L. C. \& Zhao, Y. Auxin perception and downstream events. Curr. Opin. Plant. Biol. 33, 8-14 (2016).

46. Tiwari, S. B., Hagen, G. \& Guilfoyle, T. J. Aux/IAA proteins contain a potent transcriptional repression domain. Plant Cell 16, 533-543 (2004).

47. Guo, H. \& Ecker, J. R. Plant responses to ethylene gas are mediated by SCF (EBF1/EBF2)-dependent proteolysis of EIN3 transcription factor. Cell 115, 667-677 (2003).

48. Vert, G. \& Chory, J. Downstream nuclear events in brassinosteroid signalling. Nature 441, 96-100 (2006).

49. Davière, J. M. \& Achard, P. A pivotal role of DELLAs in regulating multiple hormone signals. Mol. Plant 9, 10-20 (2016)

50. MacQuarrie, K. L., Fong, A. P., Morse, R. H. \& Tapscott, S. J. Genome-wide transcription factor binding: beyond direct target regulation. Trends Genet. 27, 141-148 (2011).

51. Brenner, W. G. \& Schmülling, T. Summarizing and exploring data of a decade of cytokinin-related transcriptomics. Front. Plant Sci. 6, 29 (2015).

52. Godoy, M. et al. Improved protein-binding microarrays for the identification of DNA-binding specificities of transcription factors. Plant J. 66, 700-711 (2011).

53. Weirauch, MatthewT. et al. Determination and inference of eukaryotic transcription factor sequence specificity. Cell 158, 1431-1443 (2014).

54. Brand, U., Fletcher, J. C., Hobe, M., Meyerowitz, E. M. \& Simon, R. Dependence of stem cell fate in Arabidopsis on a feedback loop regulated by CLV3 activity. Science 289, 617-619 (2000).

55. Mayer, K. F. et al. Role of WUSCHEL in regulating stem cell fate in the Arabidopsis shoot meristem. Cell 95, 805-815 (1998).

56. Buechel, S. et al. Role of A-type Arabidopsis response regulators in meristem maintenance and regeneration. Eur. J. Cell Biol. 89, 279-284 (2010).

57. Zhang, T. Q. et al. A two-step model for de novo activation of WUSCHEL during plant shoot regeneration. Plant Cell. 29, 1073-1083 (2017).

58. Meng, W. et al. Type-B Arabidopsis response regulators is critical to the specification of shoot stem cell niche by dual regulation of WUSCHEL. Plant Cell 29, 1357-1372 (2017).

59. Dai, X. et al. ARR12 promotes de novo shoot regeneration in Arabidopsis thaliana via activation of WUSCHEL expression. J. Integr. Plant Biol. 59, 747-758 (2017).

60. Wielopolska, A., Townley, H., Moore, I., Waterhouse, P. \& Helliwell, C. A high-throughput inducible RNAi vector for plants. Plant Biotechnol. J. 3, 583-590 (2005).

61. Brand, U., Grünewald, M., Hobe, M. \& Simon, R. Regulation of CLV3 expression by two homeobox genes in Arabidopsis. Plant Physiol. 129, 565-575 (2002).

62. Yadav, R. K., Tavakkoli, M. \& Reddy, G. V. WUSCHEL mediates stem cell homeostasis by regulating stem cell number and patterns of cell division and differentiation of stem cell progenitors. Development 137, 3581-3589 (2010).

63. To, J. P. et al. Cytokinin regulates type-A Arabidopsis response regulator activity and protein stability via two-component phosphorelay. Plant Cell 19, 3901-3914 (2007).

64. Dreher, K. A., Brown, J., Saw, R. E. \& Callis, J. The Arabidopsis Aux/IAA protein family has diversified in degradation and auxin responsiveness. Plant Cell 18, 699-714 (2006).

65. Gao, R. \& Stock, A. M. Biological insights from structures of two-component proteins. Annu. Rev. Microbiol. 63, 133-154 (2009).

66. Werner, T., Köllmer, I., Bartrina, I., Holst, K. \& Schmülling, T. New insights into the biology of cytokinin degradation. Plant Biol. 8, 371-381 (2006).

67. Hill, K. Post-translational modifications of hormone-responsive transcription factors: the next level of regulation. J. Exp. Bot. 66, 4933-4945 (2015).

68. Zürcher, E. et al. A robust and sensitive synthetic sensor to monitor the transcriptional output of the cytokinin signaling network in planta. Plant Physiol. 161, 1066-1075 (2013).

69. Bäurle, I. \& Laux, T. Regulation of WUSCHEL transcription in the stem cell niche of the Arabidopsis shoot meristem. Plant Cell 17, 2271-2280 (2005).

70. Clough, S. J. \& Bent, A. F. Floral dip: a simplified method for Agrobacteriummediated transformation of Arabidopsis thaliana. Plant J. 16, 735-743 (1998).

71. Earley, K. W. et al. Gateway-compatible vectors for plant functional genomics and proteomics. Plant J. 45, 616-629 (2006).

72. Langmead, B., Trapnell, C., Pop, M. \& Salzberg, S. L. Ultrafast and memoryefficient alignment of short DNA sequences to the human genome. Genome Biol. 10, R25 (2009).
73. Li, Q., Brown, J. B., Huang, H. \& Bickel, P. J. Measuring reproducibility of high-throughput experiments. Ann. Appl. Stat. 5, 1752-1779 (2011).

74. Heinz, S. et al. Simple combinations of lineage-determining transcription factors prime cis-regulatory elements required for macrophage and B cell identities. Mol. Cell 38, 576-589 (2010).

75. Bailey, T. L., Johnson, J., Grant, C. E. \& Noble, W. S. The MEME suite. Nucleic Acids Res. 43, W39-W49 (2015).

76. Ashburner, M. et al. Gene ontology: tool for the unification of biology. The gene ontology consortium. Nat. Genet. 25, 25-29 (2000).

77. Csardi, G. \& Nepusz, T. The igraph software package for complex network research. InterJ. Complex Syst. 1695, 1695 (2006).

78. Shannon, P. et al. Cytoscape: a software environment for integrated models of biomolecular interaction networks. Genome Res. 13, 2498-2504 (2003).

79. Kim, D. et al. TopHat2: accurate alignment of transcriptomes in the presence of insertions, deletions and gene fusions. Genome Biol. 14, R36 (2013).

80. Trapnell, C. et al. Transcript assembly and quantification by RNA-Seq reveals unannotated transcripts and isoform switching during cell differentiation. Nat. Biotechnol. 28, 511-515 (2010).

\section{Acknowledgements}

We thank Joseph Nery, Rosa Castanon, Yusuke Koga, and Huaming Chen for their excellent technical support and Drs. Mark Zander (Salk Institute), and Mathew Lewsey (La Trobe University) for critical reading of the manuscript. Dr. Shao-shan Carol Huang for alignment and analysis pipelines for bioinformatic suggestions (Salk Institute). We thank Dr. G. Venugopala Reddy (UCR) for helping the imaging experiment and providing the $p C L V 3: L h G 4 G R / p M X 6 x O P s: W U S$ transgenic line and Dr. H. Sakai for the $35 S$ : $A R R 1 D D K$ construct. This work was supported by grants from National Science Foundation (MCB-1024999 to J.R.E.) and the Gordon and Betty Moore Foundation (GBMF 3034 to J.R.E.). J.R.E. is an Investigator of the Howard Hughes Medical Institute. This work was supported by the Razavi Newman Integrative Genomics and Bioinformatics Core Facility of the Salk Institute with funding from NIH-NCI CCSG: P30 014195, and the Helmsley Trust.

\section{Author contributions}

J.R.E. and M.X. conceived the project. M.X. generated the materials and all data. M.X., H. C., L.H., R.C.O., M.N.S., and J.R.E. analyzed data. M.X. and J.R.E. wrote the manuscript that also were edited by R.C.O., L.H., M.N.S., and H.C.

\section{Additional information}

Supplementary Information accompanies this paper at https://doi.org/10.1038/s41467018-03921-6.

Competing interests: The authors declare no competing interests.

Reprints and permission information is available online at http://npg.nature.com/ reprintsandpermissions/

Publisher's note: Springer Nature remains neutral with regard to jurisdictional claims in published maps and institutional affiliations.

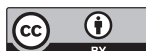

Open Access This article is licensed under a Creative Commons Attribution 4.0 International License, which permits use, sharing, adaptation, distribution and reproduction in any medium or format, as long as you give appropriate credit to the original author(s) and the source, provide a link to the Creative Commons license, and indicate if changes were made. The images or other third party material in this article are included in the article's Creative Commons license, unless indicated otherwise in a credit line to the material. If material is not included in the article's Creative Commons license and your intended use is not permitted by statutory regulation or exceeds the permitted use, you will need to obtain permission directly from the copyright holder. To view a copy of this license, visit http://creativecommons.org/ licenses/by/4.0/.

(C) The Author(s) 2018 